\title{
Stochastic Dynamics of Extended Objects in Driven Systems: I. Higher-Dimensional Currents in the Continuous Setting
}

\author{
Michael J. Catanzaro ${ }^{\mathrm{a}}$, Vladimir Y. Chernyak ${ }^{\mathrm{a}, \mathrm{b}}$, John R. Klein ${ }^{\mathrm{a}}$ \\ ${ }^{a}$ Department of Mathematics, Wayne State University, 656 W. Kirby, Detroit, MI 48202 \\ ${ }^{b}$ Department of Chemistry, Wayne State University, 5101 Cass Ave, Detroit, MI 48202
}

\begin{abstract}
The probability distributions, as well as the mean values of stochastic currents and fluxes, associated with a driven Langevin process, provide a good and topologically protected measure of how far a stochastic system is driven out of equilibrium. By viewing a Langevin process on a compact oriented manifold of arbitrary dimension $m$ as a theory of a random vector field associated with the environment, we are able to consider stochastic motion of higher-dimensional objects, which allow new observables, called higher-dimensional currents, to be introduced. These higher dimensional currents arise by counting intersections of a $k$-dimensional trajectory, produced by a evolving $(k-1)$-dimensional cycle, with a reference cross section, represented by a cycle of complimentary dimension $(m-k)$. We further express the mean fluxes in terms of the solutions of the Supersymmetric Fokker-Planck (SFP), thus generalizing the corresponding well-known expressions for the conventional currents.
\end{abstract}

\section{Introduction}

Stochastic Langevin processes appear in a wide variety of fields $[26,50,71,24,35,62]$. This is not surprising if one thinks of these as a result of elimination of fast environmental (bath) degrees of freedom, resulting in dissipative and noise terms in the reduced equations for the system $[51,26]$. The popularity of the Langevin approach in describing dynamical systems that occur in different applications can be explained in a variety of ways. The Langevin equation is simple, universal, and adequately describes the classical dynamics of an open system whose dynamical time scale is slow compared to the bath relaxation time, including the system relaxation to its steady state $[66,26]$. In the case of detailed balance, the steady state is represented by a Boltzmann distribution [75].

Stationary systems whose steady state is a true equilibrium, characterized by the absence of fluxes of any kind, as of today can be described as well as studied, at least on the conceptual level, in both the classical and quantum cases [65]. Steady states of generic or, in other words, driven systems, often referred to as non-equilibrium steady states, are much less understood, even in the case of classical open systems coupled to a fast bath, when the latter degrees of freedom can be efficiently eliminated, resulting in a Langevin equation [32]. Despite the fact that Langevin processes can be efficiently studied, e.g., by switching from the Lagrangian language of stochastic trajectories to the Euler-Hamilton picture of deterministic Fokker-Planck (FP) equations for the probability distributions $[26,35,75,66]$, theories for the steady states of driven systems are still in the developmental stage. This is not surprising since, even in the absence of noise, a driven sys- tem is described by a generic system of ODEs and its behavior can be very rich, ranging from integrable systems to systems which possess various degrees of chaos. On the other hand, studying non-equilibrium systems is really necessary, since most systems of interest are in fact driven, e.g., biological systems $[2,3,46,50,60]$, environmental systems [63], as well as various kinds of networks, including electrical power grids [24], gas supply networks [24, 25], networks describing price markets $[9,49]$, and many others $[64,58,2,63]$.

Over the past three decades a substantial effort has been put into not only studying the behavior of specific driven systems, but also in identifying a set of basic principles and laws that would govern them. In other words, such efforts amount to building an analogue of thermodynamics, often referred to as non-equilibrium thermodynamics $[30,43,40]$. A large variety of such laws, universal exact relations, and concepts have already been identified. These include various kinds of fluctuation theorems for produced work [1, 41, 13], generated heat and entropy [33, 28, 27], and similar observables, as well as their reduced counterparts, known as the Jarzynski relations [45, 44, 39, 43, 12].

A very useful concept that has been drawing more and more attention recently is that of a stochastic current, or flux $[71,64,61,32,6,14]$. It would be worth emphasizing, that although stochastic current densities appear in any driven system, to have a non-trivial flux the configuration space of a system should have non-trivial topology [15]. In fact, it should have non-contractible 1-dimensional cycles; in other words, flux has a topological nature. The topological nature of flux implies it is a topologically protected quantity, since it is based on counting the total 
number of events by the total time to obtain the rates. As outlined, e.g., in [15], a flux can be measured in two equivalent ways, by counting some kind of a winding number, i.e., how many times the system spans a given noncontractible cycle, or alternatively, by how many times a stochastic trajectory crosses a reference cross section. The equivalence between the two approaches to a measurement was established in [15], and relies on one of the most basic principles in algebraic topology of manifolds, known as Poincaré duality $[73,36]$. Currents and fluxes are also welldefined for the discrete counterpart of Langevin processes, namely continuous-time Markov Chain (MC) stochastic processes on graphs $[17,19]$. In this case, current/flux is measured by counting how many times a stochastic trajectory (Markov chain) goes over a given graph edge, with the proper sign that accounts for the direction. There is another good reason for the importance of the current/flux concept: it turns out that the probability density together with current density constitute the right set of variables for studying the long-time limit of the probability distributions. The large-deviation (Cramér) function $\mathcal{S}(\rho, \boldsymbol{J})$, referred to as the universal current density functional that describes the long-time behavior of the probability distribution function (functional) of the density $\rho$ and current density $\boldsymbol{J}$, has a simple form and can be written explicitly in the continuous as well as discrete cases, see, e.g. $[8,5,6,34,32,52,53,15]$. This fact forms the basis of a field, often referred to as the 2.5-level theory [4]. The $(\rho, \boldsymbol{J})$ description turned out to also be important in stochastic optimal control theory, leading to the so-called GaugeInvariant-Hamilton-Jacobi-Bellman (HJB) equation that extends control to cost functionals with the terms linear in velocity, as well as providing an optimization view of the celebrated HJB equation [14, 7].

On the one hand, studying the probability distributions of currents and fluxes in specific systems has shown the usefulness of the concept by providing non-trivial measures of the driven nature of the underlying stochastic dynamics. On the other hand, a large number of exact relations for generated currents in driven systems has been established, so far mostly in the discrete setting, thus making the current/flux good candidates for building a theory of non-equilibrium thermodynamics. While the fluctuation theorems for entropy production and related quantities [28, 29, 48, 38, 42, 68] are despite their non-triviality, well understood by now, and in most cases follow from comparing the probability of a stochastic trajectory with its time-reversed counterpart, the exact relations for currents and fluxes in both open [16] and closed [72] networks, turns out to be more surprising. A very interesting class of effects is related to the situation of periodic driving, when at any given time the system is in detailed balance, while the system parameters change in time in a periodic fashion, with the latter dependence being the only source of driving $[20,54]$. For this setting a variety of exact statements have been identified, including no-pumping and pumping restriction statements $[17,22]$. One of the most interest- ing findings was realizing that the fluxes are still generated in the adiabatic limit, when effects of driving become minimal, and have geometric nature, being interpreted in terms of stochastic Berry phases $[61,70,71]$. Further studies of the adiabatic low-temperature limit revealed the flux quantization effect $[17,18,19]$, i.e, in this limit the flux, defined as the number of counted events per driving protocol becomes an integer in a generic case, and rational in the presence of permanent degeneracies; the effect has been observed experimentally.

In this manuscript, we generalize the notion of a stochastic current/flux to a higher-dimensional case, thus providing new observables associated with Langevin processes in continuous spaces, that characterize in a robust, topologically protected way the extent to which a stochastic system is driven out of equilibrium. In other words we bring in topological concepts that provide a better understanding of physics of non-equilibrium phenomena, as well as new insights to non-equilibrium thermodynamics. The aforementioned generalization is very natural and in fact simple. Starting with the interpretation of a stochastic flux as an intersection index (the sum over intersection points weighted with the proper \pm 1 sign factors to account for direction) of a stochastic trajectory in a space $X$ of dimension $\operatorname{dim}(X)=m$ with a cross section, we extend our consideration to stochastic motion of higher-dimensional objects. Namely, we consider $(k-1)$-dimensional cycles that span $k$-dimensional trajectories and count their intersections with a reference cross section, represented by a cycle of complimentary dimension $(m-k)$, weighting them with the proper sign factors, resulting in the intersection index of two manifolds (a stochastic trajectory and the cross section). Summarizing, the higher-dimensional flux is an observable that associates with a $k$-dimensional stochastic trajectory its intersection index with a reference $(m-k)$-dimensional cross section per unit time. To define higher-dimensional trajectories generated in a Langevin process, one needs to do a very simple and natural thing of replacing the traditional correlation function of the random field in Eq. (2) with a more general one in Eq. (1), which immediately interprets a Langevin process as what it actually is - a theory of random vector fields, or equivalently, a theory of stochastic flows.

In the case of standard currents, the observables can be efficiently computed by switching from the Lagrangian language of stochastic trajectories to the Euler-Hamilton picture of deterministic linear PDEs for the probability distributions [17, 18, 71]. The Euler-Hamilton picture involves studying the Fokker-Planck (FP; or Kolmogorov in the mathematical literature) equations. To generalize the FPequation approach to higher-dimensional fluxes, we adopt the proposal of Tanase-Nicola and Kurchan [74], who showed on an intuitive level that the so-called Supersymmetric Fokker-Planck (SFP) equation adequately describes stochastic evolution of higher-dimensional objects. In fact, we formalize the ideas presented in [74] to develop an interpretation of the super-states $\varrho\left(x^{1}, \ldots, x^{m} ; \Theta^{1}, \ldots, \Theta^{m}\right)$ 
that depend not only on the standard set coordinates $x$, but also on a set of Grassmann (anticommuting) variables, and satisfy the SFP equation, as reduced measures that contain reduced, yet all the necessary information on the complete probability distributions $d \mathcal{P}(\eta)$ in the infinitedimensional (functional) of $(k-1)$-dimensional cycles in $X$, with the reduction being compatible with stochastic evolution. The above interpretation made it possible to obtain the main result of this manuscript for continuous modelsclosed expressions for average higher-dimensional stochastic fluxes in terms of the solutions of the SFP equations for the general time-dependent, stationary and periodicdriving cases.

We would like to emphasize that the higher-dimensional fluxes are not associated with a new class of stochastic models, but rather provide a set of new topologically protected observables, associated with the same "good old" Langevin processes. However, they give rise to a new class of discrete models, defined on CW-complexes, which are higher-dimensional generalizations of graphs. These more general discrete models, studied in the second manuscript, appear in a natural way by considering the long-time relaxation of higher-dimensional cycles in the low-temperature limit for a Langevin process, in the same way as a Markov process on a graph can be interpreted as slow relaxation between the local minima of the potential function $V(x)$, represented by the graph vertices, via rare over-the-barrier transition events, with the transition paths represented by the graph edges; actually describing a multi-state thermoactivated chemical reaction, considered within the multidimensional version of the celebrated Kramers transition state theory $[47,37]$. After deriving the CW-complex models, including evolution equations for the reduced measures (a discrete counterpart of the reduced measures for Langevin processes), we focus on the periodic driving case. The main result of the second manuscript proves there is an explicit formula for the generated flux in the adiabatic limit, which generalizes the expression for a Markov process on a graph, obtained in our earlier work [17], [18], [19], to the higher-dimensional case. To that end, we express the two ingredients of this expression, namely the solution of the higher-dimensional Kirchhoff network problem [11] and the higher-dimensional Boltzmann distribution [10], as a weighted sum over spanning trees and co-trees. These results are referred to as the higher-dimensional Kirchhoff tree and co-tree theorems, respectively. The aforementioned expression allows us to demonstrate that in the low-temperature, adiabatic limit, the generated fluxes are quantized (pumping quantization theorem). However in a generic case quantization is rational, rather than integer, which reflects higher complexity in the stochastic evolution of higher-dimensional cycles.

At this point we would like to note that although the dynamics we are dealing with in this manuscript is a standard Langevin process that is a common tool in chemical physics, the topological and higher-dimensional nature of the new observables requires a certain number of theoret- ical techniques, which are common in topology and geometry, rather than in chemical physics. Still, the problems addressed in this manuscript belong to the scope of chemical physics. Therefore, to make the manuscript accessible for the chemical physics community we have put substantial effort to describe all the concepts and techniques, involved in the derivations at least on an intuitive level, and with enough detail, so that a reader can follow the derivations without a necessity to read additional mathematical literature.

This manuscript is organized as follows. In section 2, we describe the main concepts and technical tools involved in our derivations, which are not very traditional in the chemical physics community, as well as formulate the main results presented in these two manuscripts. A detailed outline of the material presented there is given at the beginning of the section; here we just note that the main results of this manuscript for higher-dimensional fluxes in continuous stochastic systems are presented in subsections 2.5. Section 3 is devoted to the derivations of our main results in the continuous setting, formulated in subsection 2.5. In subsection 3.1 we formulate a path-integral representation for the average flux and the generating function. In subsection 3.2, we introduce our main computational tool, namely the reduced measures, and apply it to convert the path-integral representations to the Hamilton-Euler approach of fermionic super-states and SFP equations. In subsection 3.3, we provide the derivations of the final expressions for the average flux in the stationary and periodically driven cases. In section 4 , we summarize our results on the continuous setting and discuss some future possible developments/applications.

\section{Technical Introduction}

In this section, we describe the scope of this work and formulate the main results presented in the manuscript. Since the aim of this manuscript is rather technical, we will summarize here the main results and main concepts needed to formulate the aforementioned results.

The material in this section is organized as follows. Subsection 2.1 presents a very important interpretation of Langevin processes as a theory of stochastic flows generated by random vector fields. Equivalently, Langevin processes can be thought of as random walks in the space of diffeomorphisms of the configuration space $X$. In subsection 2.2, we introduce the concept of a higher dimensional cycle $\gamma$, and place an equivalence relation on the cycles to form the homology groups $H(X)$, which turn out to be abelian groups or vector spaces. Furthermore, we introduce a higher-dimensional stochastic flux as an intersection index of a stochastic trajectory $\eta$ with a reference cross section $\alpha$, describe the flux in terms of homology, and introduce the closely related concept of Poincaré duality. To provide some intuition and insight for higher dimensional fluxes, we present several simple examples of deterministic flows that generate non-zero higher dimensional 
fluxes in subsection 2.3. In subsection 2.4, we provide the necessary facts about supersymmetric stochastic dynamics, including the Supersymmetric Fokker-Planck (SFP) equation, related geometric structures, and de Rham cohomology $H_{\mathrm{DR}}(X)$ - the residence of the equivalence classes $[\psi]$ of the fermion (super)states $\psi$. In section 2.5, we revisit Poincaré duality and present its formulation as an equivalence between homology and cohomology in complimentary dimensions. This allows us to formulate and present the main results of this manuscript, related to higherdimensional currents in the continuous setting. Namely, we provide closed expressions for average values of stochastic fluxes in terms of solutions of the SFP equation, including the cases of general, stationary, and periodic potential driving.

\subsection{Langevin Processes as Random Walks in Diffeomor- phism Groups and Regularization}

We reiterate that we are dealing with Langevin processes on a smooth oriented compact manifold $X$ of dimension $\operatorname{dim} X=m$. The compactness requirement can be relaxed, when necessary, at the cost of adding some technical details.

As described above, standard empirical currents can be viewed as a topological observable for a Langevin process in a manifold $X$ that associates to each stochastic trajectory its homology class. A Langevin process with Gaussian Markovian noise can be represented by a stochastic equation

$$
\begin{gathered}
\dot{x}^{j}=u^{j}(x, t)+\xi^{j}(x, t), \\
\left\langle\xi^{j}(x, t)\right\rangle=0, \\
\left\langle\xi^{i}(x, t) \xi^{j}\left(y, t^{\prime}\right)\right\rangle=2 \kappa G^{i j}(x, y) \delta\left(t-t^{\prime}\right),
\end{gathered}
$$

where $\xi(t)$ is random vector field with Gaussian Markovian statistics, fully described by the correlation function $G$, and $\kappa=\beta^{-1}=\left(k_{B} T\right)^{-1}$ controls the noise strength. The tensor field $g^{i j}(x)=G^{i j}(x, x)$ defines a non-negative metric in $X$ (strictly speaking a scalar product in the cotangent bundle). We will assume that $g$ is non-degenerate, which implies that it is positive definite. This means that our configuration space $X$ is equipped with a Riemannian metric $g_{i j}(x)$, defined by the condition $g_{i k}(x) g^{k j}(x)=\delta_{i}^{j}$, where $\delta_{i}^{j}$ is the Kronecker delta, and hereafter we imply summation over repeating indices. At this point, it is worth noting that in describing a Langevin process Eq. (1) is usually replaced with

$$
\begin{gathered}
\dot{x}=u(x, t)+\xi(x, t), \\
\left\langle\xi^{j}(x, t)\right\rangle=0, \\
\left\langle\xi^{i}(x, t) \xi^{j}\left(x, t^{\prime}\right)\right\rangle=2 \kappa g^{i j}(x) \delta\left(t-t^{\prime}\right),
\end{gathered}
$$

which is consistent with Eq. (1). The reason is that in a standard set-up a Langevin process is studied on the level of stochastic dynamics of points in the configuration space $X$. Therefore, due to the Markovian, i.e., the $\delta$ correlated in time, nature of a Langevin process, the correlation properties of the random vector field $\xi(x)$ at the same point of the configuration space $X$ only are relevant for the standard set-up. In what follows we will be studying the evolution of higher-dimensional objects, so that the complete information on the underlying Langevin process, given by Eq. (1) is required. Stated differently, this implies that the stochastic dynamics of higher-dimensional objects contains more detailed information on the underlying Langevin process.

Most importantly, the r.h.s. of Eq. (1) determines a flow and, therefore, a random Markovian walk in the group $\operatorname{Diff}(X)$ of diffeomorphisms of $X$. This should be understood as follows. Vector fields on $X$ can be naturally interpreted as infinitesimal diffeomorphisms, i.e., the Lie algebra $\operatorname{Vect}(X)$ of vector fields in $X$ can be viewed as the Lie algebra $\mathfrak{a}(\operatorname{Diff}(X))$ associated with the group $\operatorname{Diff}(X)$ of diffeomorphisms of $X$. Furthermore, since vector fields represent first-order differential operators, a two-fold tensor product of vector fields represents a second-order differential operator. The deterministic (advection term) vector field $u \in \operatorname{Vect}(X)$ can be considered as the element of the relevant algebra $\operatorname{Vect}(X)=\mathfrak{a}(\operatorname{Diff}(X))$, whereas we can treat the noise correlation function as a symmetric element $G \in \operatorname{Vect}(X) \otimes \operatorname{Vect}(X)$. This allows the generalized Fokker-Planck (FP) operator

$$
\hat{\mathcal{L}}=\kappa G+u
$$

to be introduced as an element of the universal enveloping algebra $U \operatorname{Vect}(X)=U \mathfrak{a}(\operatorname{Diff}(X))$ of the Lie algebra $\operatorname{Vect}(X)$ of vector fields in $X{ }^{1}$ Therefore, we can view a Langevin process as a left-invariant random walk in the group $\operatorname{Diff}(X)$.

Random processes, whose Euler (Hamilton) representation is described by Eq. (3), have been considered for the case of a finite-dimensional Lie group $H$, with the corresponding finite-dimensional $\mathfrak{a}(H)$, in the context of the Lyapunov exponent statistics in chaotic systems for $H=$ $S L(n ; \mathbb{R})$ with the corresponding $\mathfrak{a}(S L(n ; \mathbb{R}))=\operatorname{sl}(n ; \mathbb{R})$. This includes the passive scalar turbulence model [23], where chaotic behavior of the linear infinitesimal deviations is modeled by stochastic behavior, as well as linear [67] and nonlinear $[55,56]$ responses for weak-noise $\kappa \rightarrow 0$ geodesic flows in Riemann surfaces of genus $g \geq 2$ with constant negative curvature that corresponds to the case of $H=S L(2 ; \mathbb{R})$.

It is the dynamical symmetry, expressed by Eq. (3), i.e., the fact that the relevant FP operator $\hat{\mathcal{L}} \in U \mathfrak{a}(H)$ belongs to the universal enveloping algebra associated with

\footnotetext{
${ }^{1}$ The universal enveloping algebra $U \mathfrak{h}$ of a Lie algebra $\mathfrak{h}$ is $T \mathfrak{h}=$ $\sum_{p=0}^{\infty} \mathfrak{h}^{\otimes p}=\mathbb{R} \oplus \mathfrak{h} \oplus(\mathfrak{h} \otimes \mathfrak{h}) \oplus \ldots$ with the relations $a \otimes b-b \otimes a-[a, b]=$ 0 for $a, b \in \mathfrak{h}[69]$. The natural morphism $T \mathfrak{h} \rightarrow U \mathfrak{h}$ generates maps $\mathfrak{h} \rightarrow U \mathfrak{h}$ and $\mathfrak{h} \otimes \mathfrak{h} \rightarrow U \mathfrak{h}$ so that with a minimal abuse of notation we can view $\mathfrak{h} \subset U \mathfrak{h}$ and $\mathfrak{h} \otimes \mathfrak{h} \subset U \mathfrak{h}$
} 
the Lie algebra $\mathfrak{a}(H)$ of the dynamical symmetry group $H$ that makes the aforementioned problems to be tractable on the analytical level $[55,56]$, e.g., by decomposing the space of distributions into irreducible representations and making use of the fact that in each representation the evolution occurs independently (which is due to the dynamical symmetry).

The stochastic equation (1) should be properly regularized. We choose a time regularization by representing the random vector field $\xi(t)$ as a piece-wise constant function of time. Specifically we split the time interval $[0, t]$ into short segments of length $\varepsilon$ and assume the random vector field to be time-independent on each segment with the correlation function

$$
\left\langle\xi^{j}(x)\right\rangle=0, \quad\left\langle\xi^{i}(x) \xi^{j}(y)\right\rangle=2 \kappa \varepsilon^{-1} G^{i j}(x, y),
$$

with no correlations between the random vector fields on different segments.

Spatial regularization can be achieved by restricting the random field $\xi$ to a finite-dimensional vector subspace $\mathcal{U}$ of the space of smooth vector fields $\operatorname{Vect}(X)$ together with a positively defined scalar product in $\mathcal{U}$, which naturally defines Gaussian fluctuations of the allowed vector fields $\xi \in \mathcal{U}$. Obviously for a regularized correlation function we have $G \in \mathcal{U} \otimes \mathcal{U} \in \operatorname{Vect}(X) \otimes \operatorname{Vect}(X)$.

\subsection{Higher-Dimensional Stochastic Currents and Poincaré Duality}

Conventional empirical currents have been identified [15] as topologically protected observables. The approach is based on the notion of cycles associated with stochastic trajectories and measurements. In this manuscript, a $k$ dimensional cycle in $X$ is understood as a map (smooth, continuous, or piece-wise smooth depending on the context) map $\gamma: K \rightarrow X$ from a smooth oriented compact $k$-dimensional manifold $K$ to $X$. The cycles can be added by taking a union, i.e., for $\gamma: K \rightarrow X$ and $\gamma^{\prime}: K^{\prime} \rightarrow X$ we define $\gamma+\gamma^{\prime}: K \sqcup K^{\prime} \rightarrow X$. We also define $-\gamma$ by just reversing the orientation of $K$. Therefore the cycles form an abelian group. We call $\gamma \sim 0$ if there is a map (bordism) $\chi: M \rightarrow X$ for a smooth $(k+1)$-dimensional manifold $M$ with the border $\partial M=K$ so that its restriction to the border reproduces $\gamma$, i.e., $\left.\chi\right|_{\partial M}=\gamma$. We call $\gamma \sim \gamma^{\prime}$ when $\gamma-\gamma^{\prime} \sim 0$. The abelian group obtained from the group of $k$-dimensional cycles by considering the equivalence classes $[\gamma]$ with respect to the described above equivalence relation (referred to as bordism equivalence) is called the oriented bordism homology group [59] of $X$ and will be denoted $H_{k}(X)$. In dealing with empirical currents we will work with the homology with real coefficients defined as vector spaces $H_{k}(X ; \mathbb{R})=H_{k}(X) \otimes_{\mathbb{Z}} \mathbb{R}^{2}$

\footnotetext{
${ }^{2}$ The theory of higher-dimensional empirical currents can be also developed by understanding the cycles $\gamma$ as so-called singular cycles [73]. In this case the currents are generated by stochastic motion of
}

In the standard case of a stochastic trajectory represented by a map $\eta:[0, t] \rightarrow X$, as argued in [15], the analysis can be restricted to closed trajectories represented by 1-cycles $\eta: S^{1} \rightarrow X$, so that the equivalence class $t^{-1}[\eta] \in H_{1}(X ; \mathbb{R})$ represents the contribution of a stochastic trajectory $\eta$ to the empirical current $\omega \in H_{1}(X ; \mathbb{R})$. As outlined in [15], Poincáre duality allows for an equivalent interpretation of the empirical current as a set of components represented by a set of fluxes over a set of $(m-1)$-dimensional cycles $\alpha: K \rightarrow X$, where the intersection index $t^{-1}[\eta] \cdot[\alpha]$ is viewed as the contribution of the stochastic trajectory to the component $\omega_{\alpha} \in \mathbb{R}$ of the empirical current $\omega .^{3}$

In this manuscript we extend the notion of empirical currents to higher dimensions, i.e., we introduce currents generated in the full homology $H_{\bullet}(X ; \mathbb{R})$ with $\bullet=1,2, \ldots, m$, so that the case $\bullet=1$ reproduces the standard empirical currents considered in [15]. This generalization rests on the interpretation of a Langevin process as a random walk in $\operatorname{Diff}(X)$, as described in subsection 2.1. To put it on a formal grounds, we denote by $f(\xi):[0, t] \rightarrow \operatorname{Diff}(X)$ a stochastic trajectory that starts at the identity diffeomorphism $\operatorname{id}_{X} \in \operatorname{Diff}(X)$ and is generated by the noise realization $\xi:[0, t] \rightarrow \operatorname{Vect}(X)$ according to the Langevin equation [Eq. (1)]. Note that for the time regularization described in subsection 2.1 the trajectory $f$ is continuous and piece-wise smooth.

Given a stochastic trajectory $f(\xi)$ we can associate with any $n$-dimensional cycle $\gamma: N \rightarrow X$, considered as an initial condition, an $(n+1)$-dimensional chain, represented by $\eta(\xi, \gamma):[0, t] \times N \rightarrow X$, with $\eta(\xi, \gamma)(\tau, y)=f(\xi ; \tau)(\gamma(y))$, which can be viewed as a stochastic trajectory in the space $X^{N}$ of smooth maps $N \rightarrow X{ }^{4}$ If this trajectory were periodic (with period $t$ ), it would be represented by a cycle $\eta(\xi, \gamma): S^{1} \times N \rightarrow X$, whose homology class $t^{-1}[\eta] \in H_{n+1}(X ; \mathbb{R})$ will represent the contribution of the stochastic trajectory $\eta$ to the higher-dimensional current $\omega \in H_{n+1}(X ; \mathbb{R})$, in full analogy with the conventional $n=0$ case. In the latter case, a stochastic trajectory, which generally has a boundary, can be turned into a closed trajectory by connecting its ends with a geodesic line, which does not affect the long-time asymptotic of the current distributions [15]. A generalization of such closing procedure in the higher-dimensional case does not look straightforward. It will be addressed in some detail

singular, rather than oriented bordism, cycles, and $H_{k}(X)$ should be understood as singular homology [73]. As we will see, the long-time statistics of the generated currents does not depend on the particular choice of the cycle type. This relies on the fact that the natural morphism from bordism homology to singular homology becomes an equivalence over real coefficients. For this reason we are also using the notation $H_{\bullet}(X)$ for oriented bordism homology, which is more common for singular case, instead of the standard $\Omega_{\bullet}(X)$.

${ }^{3}$ In particular this means that the current components are defined as $\omega_{\alpha}=\omega \cdot[\alpha]$. We also reiterate that the intersection index is welldefined on the level of equivalence classes.

${ }^{4}$ This correspondence is actually due to a natural action $\operatorname{Diff}(X) \times$ $X^{N} \rightarrow X^{N}$ of the diffeomorphism group in the space of cycles. 
in subsection 3.1. Here we will present an intuitive picture based on the notion of empirical current components.

To this end, we introduce a $k$-dimensional cycle $\alpha$ : $K \rightarrow X$ with $k=m-(n+1)$ and introduce the intersection index $\eta \cdot \alpha$. An attempt can be made in a standard way: we slightly perturb the piece-wise smooth trajectory $\eta:[0, t] \rightarrow X^{N}$ to obtain a smooth map $\tilde{\eta}:[0, t] \times N \rightarrow X$, we further slightly perturb $\tilde{\eta}$ and $\alpha$ to achieve their transversal intersection in a finite number of points. We define the intersection index $\eta \cdot \alpha$ in a standard transversality-based way as a sum over the intersection points weighted with the proper \pm 1 sign factors determined by the orientations. If the trajectory $\eta$ was periodic, then $\eta$ would represent an $(n+1)$-dimensional cycle and the intersection index $\eta \cdot \alpha$ would be well defined, in particular it would not depend on the adjustments needed to achieve trasnversality, as described above. Later in the manuscript we will show that in the realistic case of nonperiodic trajectories the dependence on the adjustments vanishes statistically in the long-time $t \rightarrow \infty$ limit, which implies that the intersection index is well-defined statistically in the relevant for us $t \rightarrow \infty$ limit. Such a situation will be also described as that statistically the path integration is restricted to almost closed trajectories. Having said that, to set the stage we just pretend that $\eta \cdot \alpha$ is a welldefined quantity. Under such an assumption we can come up with a simple and intuitive path-integral expression for the generating function $Z\left(\lambda_{\alpha}\right)$ that fully characterizes the probability distribution function for the $\omega_{\alpha}$ component of the higher-dimensional current $\omega \in H_{n+1}(X ; \mathbb{R})$ :

$$
Z\left(\lambda_{\alpha} ; t\right)=\int \mathcal{D} \xi e^{-\kappa^{-1} S(\xi)+\lambda_{\alpha} \eta(\xi, \gamma) \cdot \alpha},
$$

where $S(\xi)$ is a quadratic in $\xi$ action that produces the correlation functions of the random vector field, given by Eq. (1), and the normalization factor is assumed to be absorbed in the measure $\mathcal{D} \xi$. Note that the path integral in Eq. (5) should be regularized as described in subsection 2.1.

The above arguments can be formalized as follows. Denote by $\omega(\gamma, \alpha ; t)$ the average value of the flux through the cross section cycle $\alpha: K \rightarrow X$ produced over time by stochastic trajectories that start with the cycle $\gamma$ : $N \rightarrow X$. Averaging can be in principle performed using the probability distribution function of the flux that corresponds to the generating function, given by Eq. (5). Let $k=\operatorname{dim}(N)+1$, and $m=\operatorname{dim}(X)$. Then $\operatorname{dim}(K)=m-k$. Verify that the following holds: for $t \rightarrow \infty$ the average flux does not depend on $t$ and depends on $\gamma$ and $\alpha$ through their homology classes $[\gamma]$ and $[\alpha]$, respectively. This creates a bilinear map $J: H_{k-1}(X) \otimes H_{m-k}(X) \rightarrow \mathbb{R}$. On the other hand the intersection index generates a bilinear map Int : $H_{k}(X) \otimes H_{m-k}(X) \rightarrow \mathbb{Z}$, which is known to be a non-degenerate over $\mathbb{R}$. This is one of possible formulations of Poincaré duality [73]. Due to non-degenerate nature of the Poincaré pairing, there is a unique linear map $\omega_{k}: H_{k-1}(X) \rightarrow H_{k}(X ; \mathbb{R})$, hereafter referred to as the flux map so that $J([\gamma] \otimes[\alpha])=\operatorname{Int}\left(\omega_{k}([\gamma]) \otimes[\alpha]\right)$ for any $[\gamma]$ and $[\alpha]$.

\subsection{Examples of Higher-Dimensional Currents and Fluxes, Generated by Deterministic Flows}

As explained in some detail in the previous subsection, a higher-dimensional stochastic flux is a new observable associated with a Langevin process, given by the intersection index of a higher-dimensional trajectory, produced by motion of a higher-dimensional cycle, with a cross section represented by another cycle of complimentary dimension to the trajectory dimension. Since a stochastic trajectory is in fact a deterministic trajectory that corresponds to some given realization of a stochastic flow, to understand the nature of such an observable one needs to understand how the flux is generated in the case of a deterministic flow. Switching to a stochastic setting is conceptually simple: one just needs to perform averaging over (stochastic) realizations of the flow with a proper probability measure. In the standard set-up of points moving to form 1-dimensional trajectories, the picture of the flux as an intersection index of a trajectory with a cross section is very intuitive. In the higher-dimensional case the picture is much less intuitive. Therefore, before we move further, we consider in this section some simple, yet nontrivial examples of deterministic flows and study the higher dimensional currents/fluxes, generated by the aforementioned flows.

Our first example deals with a simple flow in a torus $X=T^{2}=S^{1} \times S^{1}$. Using the natural coordinates $-\pi \leq$ $\theta_{1}, \theta_{2} \leq \pi$, the flow is defined by a differential equation

$$
\frac{d \theta_{1}}{d t}=u, \quad \frac{d \theta_{2}}{d t}=0
$$

If we monitor the motion of points we can choose the cross section to be a 1-dimensional cycle $\alpha: S^{1} \rightarrow S^{1} \times S^{1}$ with $\alpha_{1}(\theta)=(\theta, 0)$ or $\alpha_{2}(\theta)=(0, \theta)$. A particle's trajectory generated by the flow of Eq. (6) is periodic; during the period $t=2 \pi / u$ it will not cross the cycle $\alpha_{1}$ and cross $\alpha_{2}$ once, producing the fluxes (intersection index per unit time) $\omega_{1}=0$, and $\omega_{2}=u(2 \pi)$. Alternatively, we can represent the flux associated with the flow as a 2dimensional vector $\boldsymbol{\omega}=\left(\omega_{1}, \omega_{2}\right)$, with the dimension 2 reflecting the two independent 1-dimensional cycles in the torus $T^{2}$. We can also look at the motion of 1-dimensional cycles $\gamma: S^{1} \rightarrow S^{1} \times S^{1}$, with $\gamma_{1}=\alpha_{1}$ and $\gamma_{2}=\alpha_{2}$. Since trajectories produced by motion of 1-cycles are 2dimensional, cross sections should be 0-dimensional; so we can choose a cross section to be a point, say $(\pi / 2, \pi / 2)$. The trajectory that starts with $\gamma_{2}$ covers the whole torus during the time period $t=2 \pi / u$ producing one intersection, which corresponds to the flux $\omega\left(\gamma_{2}\right)=u / 2 \pi$. The cycle $\gamma_{1}$ moves along itself, so that there are no intersection and $\omega\left(\gamma_{1}\right)=0$. This example clearly demonstrates that the multidimensional flux depends on the moving cycle $\gamma$, actually on its homology class $[\gamma]$. This can be summarized 
in terms of the current/flux maps $\omega_{1}: H_{0}(X) \rightarrow H_{1}(X ; \mathbb{R})$ and $\omega_{2}: H_{1}(X) \rightarrow H_{2}(X ; \mathbb{R})$ as follows. Appreciating the fact that

$$
\begin{aligned}
& H_{0}\left(T^{2}\right)=\mathbb{Z} \\
& H_{1}\left(T^{2}\right)=\mathbb{Z} \oplus \mathbb{Z} \\
& H_{2}\left(T^{2}\right)=\mathbb{Z},
\end{aligned}
$$

as well as $\left[\gamma_{1}\right]=1 \oplus 0$ and $\left[\gamma_{2}\right]=0 \oplus 1$ form a basis set in $H_{1}\left(T^{2}\right)=\mathbb{Z} \oplus \mathbb{Z}$, we can write $\omega_{1}(r)=r u(2 \pi)^{-1} \oplus 0$, $\omega_{2}(r \oplus s)=s u(2 \pi)^{-1}$, with $r, s \in \mathbb{Z}$.

Our next example is a flow in a 3-dimensional torus $X=T^{3}=S^{1} \times S^{1} \times S^{1}$. Using the natural coordinates $-\pi \leq \theta_{1}, \theta_{2}, \theta_{3} \leq \pi$, the flow is defined by a differential equation

$$
\frac{d \theta_{1}}{d t}=u, \quad \frac{d \theta_{2}}{d t}=0, \quad \frac{d \theta_{3}}{d t}=0
$$

This example can be analyzed in a manner, similar to how its low-dimensional counterpart was studied above; therefore, details will be omitted. Consider for $X$ the cycles $\mu:\{*\} \rightarrow X, \gamma_{a}: S^{1} \rightarrow X, \alpha_{a}: S^{1} \times S^{1} \rightarrow X$, with $a=1,2,3$, and $\nu: T^{3} \rightarrow X$, of dimensions $0,1,2$, and 3 , respectively, that form the basis set in homology. Namely $\mu(*)=* \in X, \nu=\operatorname{id}_{X} ; \gamma_{a}$ embeds $S^{1}$ in $T^{3}$ along the $a$-th components of $S^{1}$ in $T^{3}$, whereas $\alpha_{a}$ embeds $T^{2}$ in $T^{3}$, missing the $a$-th component. We have for intersection indices $[\mu] \cdot[\nu]=1,\left[\gamma_{a}\right] \cdot\left[\alpha_{b}\right]=\delta_{a b}$. The flux maps are non-trivial in all dimensions; after a simple and transparent computation we arrive at

$$
\begin{aligned}
\omega_{1}(s[\mu]) & =s\left[\gamma_{1}\right], \\
\omega_{2}\left(r\left[\gamma_{1}\right]+l\left[\gamma_{2}\right]+s\left[\gamma_{3}\right]\right) & =u(2 \pi)^{-1}\left(l\left[\alpha_{3}\right]+s\left[\alpha_{2}\right]\right), \\
\omega_{3}\left(r\left[\alpha_{1}\right]+l\left[\alpha_{2}\right]+s\left[\alpha_{3}\right]\right) & =r u(2 \pi)^{-1}[\nu] .
\end{aligned}
$$

The examples considered in this section so far may create the misleading impression that higher-dimensional currents do not provide additional information on the driven nature of the underlying dynamics, since their properties are contained in the standard 1-dimensional currents, the latter describing motion of points. Therefore, we consider a flow on a 5-dimensional manifold $X=S^{3} \times S^{2}$ (which is simply connected, i.e., does not have non-contractible 1-dimensional cycles, and thus does not have standard 1dimensional fluxes) and demonstrate that the flow produces a 3-dimensional current/flux, generated by directed motion of a non-contractible 2-dimensional cycle. First, we will represent the 3 -dimensional sphere as $S^{3}=S U(2)$, using a standard assertion

$$
g\left(n_{0}, \boldsymbol{n}\right)=n_{0} \sigma_{0}+i \boldsymbol{n} \cdot \boldsymbol{\sigma}, \quad n_{0}^{2}+\boldsymbol{n}^{2}=1,
$$

where $\sigma_{0}$ and $\boldsymbol{\sigma}=\left(\sigma_{1}, \sigma_{2}, \sigma_{3}\right)$ are the unit and Pauli $2 \times 2$ matrices, respectively. The flow on $X=S U(2) \times S^{2}$ is determined by the following differential equation for $(g, \boldsymbol{n}) \in S U(2) \times S^{2}$

$$
\frac{d g}{d t}=i k(\boldsymbol{n} \cdot \boldsymbol{\sigma}) g, \quad \frac{d \boldsymbol{n}}{d t}=0,
$$

with $k>0$ being some arbitrary rate constant. We consider motion of a 2-dimensional cycle $\gamma: S^{2} \rightarrow S U(2) \times S^{2}$, defined by $\gamma(\boldsymbol{n})=(1, \boldsymbol{n})$ and considered as the initial condition, with $1 \in S U(2)$, being the unit element in $S U(2)$ represented by $\sigma_{0}$. The flow (in this case deterministic), defined by Eq. (10) produces a trajectory $\eta: \mathbb{R} \times S^{2} \rightarrow S U(2) \times S^{2}$. Since Eq. (10) can be easily solved analytically, we arrive at the following explicit expression for the trajectory that starts with $\gamma$

$$
\begin{aligned}
\eta(t, \boldsymbol{n}) & =(\exp (i k t(\boldsymbol{n} \cdot \boldsymbol{\sigma})), \boldsymbol{n}) \\
& =(\cos (k t)+i(\boldsymbol{n} \cdot \boldsymbol{\sigma}) \sin (k t), \boldsymbol{n}) .
\end{aligned}
$$

To compute the 3 -dimensional flux produced by the flow over time $t$, we consider the intersection index of the trajectory $\eta$ with a cross section, represented by a 2-dimensional cycle $\alpha: S^{2} \rightarrow S U(2) \times S^{2}$, defined by $\alpha(\boldsymbol{n})=\left(i \sigma_{3}, \boldsymbol{n}\right)$. To simplify the analysis, note that the considered trajectory is periodic with the period $2 \pi$ with respect to the dimensionless time $\tau=k t$, and consider the intersection of $\eta$ with $\alpha$ restricted to the time period $-\pi \leq \tau \leq \pi$. Defining intersection points as pairs of points $(\tau, \boldsymbol{n}) \in \mathbb{R} \times S^{2}$ and $\boldsymbol{n}^{\prime} \in S^{2}$, so that $\eta(\tau, \boldsymbol{n})=\alpha\left(\boldsymbol{n}^{\prime}\right)$, the latter representing the intersection condition, we find two intersection points for a time period, the "north" intersection $\tau=\pi / 2, \boldsymbol{n}=\boldsymbol{n}^{\prime}=(0,0,1)$ and the "south" one $\tau=-\pi / 2$, $\boldsymbol{n}=\boldsymbol{n}^{\prime}=(0,0,-1)$. Both intersections occur at the same point $i \sigma_{3} \in S U(2)$. To compute the intersection index we need to weight each intersection with the local intersection index \pm 1 , obtained in the following way. Choose orientations on $\mathbb{R} \times S^{2}, S^{2}$, and $S U(2) \times S^{2}$. Consider oriented basis sets $\left(\boldsymbol{e}_{0}, \boldsymbol{e}_{1}, \boldsymbol{e}_{2}\right)$ and $\left(\boldsymbol{e}_{1}^{\prime}, \boldsymbol{e}_{2}^{\prime}\right)$ at the tangent spaces at $(\tau, \boldsymbol{n}) \in \mathbb{R} \times S^{2}$ and $\boldsymbol{n} \in S^{2}$, respectively, that represent the intersection. Applying the differentials $d \eta$ and $d \alpha$ to the above basis sets we obtain $3+2=5$ vectors that form a basis set in the tangent space at the intersection point $\left(i \sigma_{3}, \boldsymbol{n}\right) \in S U(2) \times S^{2}$. If the obtained basis set has the same orientation as a one that describes the chosen orientation on $S U(2) \times S^{2}$ the local index is set to 1 ; if otherwise, it is set to -1 . A simple computation presented in Appendix A shows that the local indices of both intersection points are the same, so that the contribution of a time period to the intersection index $[\eta] \cdot[\alpha]$ is 2 . This means that the intersection index during time $t$ is approximately equal to $2 k t /(2 \pi) .{ }^{5}$ This corresponds to the value of the 3 -dimensional flux $\omega=2 k /(2 \pi)$.

This last example has an interpretation in terms of the flux map as follows. For $X=S^{3} \times S^{2}$, we have

$$
H_{0}(X) \cong H_{2}(X) \cong H_{3}(X) \cong H_{5}(X) \cong \mathbb{Z},
$$

with $H_{2}(X)$ and $H_{3}(X)$ generated by $[\alpha]$ and $[\gamma]$, respectively. The only nontrivial flux map $\omega_{3}: H_{2}(X) \rightarrow H_{3}(X)$ has a form $\omega_{3}(s[\gamma])=2 \operatorname{sk}(2 \pi)^{-1}[\alpha]$.

\footnotetext{
${ }^{5}$ As briefly explained above approximately means the following. If the trajectory is periodic, i.e., in this case $k t=2 \pi n$, the equality is exact. Still for long times $k t \gg 2 \pi$ the equality holds approximately, with the relative error $\sim(k t)^{-1}$, even if the trajectory is not periodic, the latter being a typical case.
} 


\subsection{Supersymmetric Fokker-Planck Equation: Fermions and Differential Forms}

It has been outlined in [74] that the Supersymmetric Fokker-Planck (SFP) equation describes stochastic motion of higher-dimensional objects, such as $n$-dimensional surfaces in the configuration space $X$. In this subsection, we present a brief review of the SFP equation, connect the fermion states to differential forms and discuss a particlehole symmetry that, being formulated using the language of differential forms, reproduces the Hodge star transformation [36] that can be viewed as the supersymmetric counterpart of the Poincáre duality.

We reiterate that our configuration space $X$ is an oriented compact smooth manifold of dimension $\operatorname{dim} X=m$ and start with introducing $n$-fermion states $\psi^{(n)}$

$$
\psi^{(n)}(x, \Theta)=\psi_{i_{1} \cdots i_{n}}^{(n)}(x) \Theta^{i_{1}} \cdots \Theta^{i_{n}},
$$

where $\Theta^{j}$ with $j=1, \ldots, m$ are Grassmann anticommuting variables, i.e. $\Theta^{j} \Theta^{i}=-\Theta^{i} \Theta^{j}$, and therefore $\left(\Theta^{i}\right)^{2}=0$. The vector space of $n$-fermion states is denoted by $A^{n}(X)$. Obviously, $A^{n}(X) \cong 0$ for $n>m$. Combining fermion states with all possible numbers of fermions we obtain a graded supercommutative algebra [57] denoted by $A^{\bullet}(X)$, whose elements (states)

$$
\begin{aligned}
\psi(x, \Theta) & =\sum_{n=1}^{m} \psi^{(n)}(x, \Theta) \\
& =\sum_{n=1}^{m} \psi_{i_{1} \cdots i_{n}}^{(n)}(x) \Theta^{i_{1}} \cdots \Theta^{i_{n}}
\end{aligned}
$$

are represented by superpositions of $m$-fermion states. ${ }^{6}$ We will also use the "bullet" as a dummy index variable $\bullet=0,1, \ldots, m$.

By replacing the Grassmann variables $\Theta^{i}$ with the differential symbols $d x^{i}$ we can recast Eq. (14) as

$$
\begin{aligned}
\psi^{(n)}(x, \Theta) & =\psi_{i_{1} \cdots i_{n}}^{(n)}(x) \Theta^{i_{1}} \cdots \Theta^{i_{n}} \\
& =\psi_{i_{1} \cdots i_{n}}^{(n)}(x) d x^{i_{1}} \wedge \cdots \wedge d x^{i_{n}} \\
& =\psi^{(n)}(x, d x),
\end{aligned}
$$

where the wedge product symbol emphasizes the anticommutative property of multiplication of the differential symbols. Most importantly, Eq. (16) identifies $n$-fermion states as rank $n$ differential forms on $X$, and therefore the fermion states inherit all properties of differential forms. The most relevant properties for us are: external differential, pull-back construction, ability to integrate an $n$ fermion sate over an $n$-cycle or $n$-chain, and Stokes' theorem.

\footnotetext{
${ }^{6}$ Using formal language, by introducing the fermion superposition states we constructed a $\mathbb{Z}_{2}$-graded smooth manifold (often referred to as a supermanifold), associated with the cotangent bundle over its bosonic substrate $X$. In this context the superposition states in Eq. (15) are referred to as the functions in the supermanifold (see, e.g., [57] for the details).
}

Following [74] we introduce an operator $\mathcal{Q}: A^{\bullet}(X) \rightarrow$ $A^{\bullet+1}(X)$ by $\mathcal{Q}=\Theta^{j} \partial_{j}$, where hereafter we use the notation $\partial_{j}=\partial / \partial x^{j}$ and $\bar{\partial}^{j}=\partial / \partial \Theta^{j}$ (acting from the left). In the language of differential forms it is known as the exterior differential operator $d: A^{\bullet}(X) \rightarrow A^{\bullet+1}(X)$. Obviously $\mathcal{Q}^{2}=0$. A fermion state of the form $\mathcal{Q} \psi$ is called exact, and a state $\psi$ with $\mathcal{Q} \psi=0$ is called closed. Obviously an exact state is closed. Denoting by $B^{\bullet}(X)$ and $Z^{\bullet}(X)$ the subspaces of the exact and closed states, respectively, the latter relation reads $B^{\bullet}(X) \subset Z^{\bullet}(X) \subset A^{\bullet}(X)$. The related quotient spaces $H_{\mathrm{DR}}^{\bullet}(X)=Z^{\bullet}(X) / B^{\bullet}(X)$ are known as the de Rham cohomology of $X$, and are finitedimensional in the relevant case of compact $X$ [36]. For a closed state $\psi \in Z^{\bullet}(X)$ its image in $H_{\mathrm{DR}}^{\bullet}(X)$ is denoted $[\psi] \in H_{\mathrm{DR}}^{\bullet}(X)$ and referred to as a (de Rham) cohomology class. We also associate with a vector field $\xi \in \operatorname{Vect}(X)$ an operator $i_{\xi}: A^{\bullet}(X) \rightarrow A^{\bullet-1}(X)$, called the inner derivative, and defined by $i_{\xi}=\xi^{j} \bar{\partial}_{j}$.

A pull-back construction associates with a smooth map $g: Y \rightarrow X$ a linear map $g^{*}: A^{\bullet}(X) \rightarrow A^{\bullet}(Y)$ that "pulls" the fermion states on $X$ back to form the corresponding states on $Y$. The pull-back that commutes with the exterior differential, i.e., $g^{*} \mathcal{Q}_{X}=\mathcal{Q}_{Y} g^{*}$ so the rank is presevered, is defined as

$$
\begin{aligned}
& \left(g^{*} \psi^{(n)}\right)_{j_{1} \cdots j_{n}}(y) \vartheta^{j_{1}} \cdots \vartheta^{j_{n}} \\
& \quad=\psi_{i_{1} \cdots i_{n}}^{(n)}(g(y)) \frac{\partial g^{i_{1}}(y)}{\partial y^{j_{1}}} \vartheta^{j_{1}} \cdots \frac{\partial g^{i_{n}}(y)}{\partial y^{j_{n}}} \vartheta^{j_{n}},
\end{aligned}
$$

which reflects the following natural transformation from the variables $(y, \vartheta)$ in $Y$ to the variables $(x, \Theta)$ in $X$.

$$
x=g(y), \quad \Theta^{i}=\left(\partial g^{i}(y) / \partial y^{j}\right) \vartheta^{j} .
$$

A fermion state $\psi \in A^{\bullet}(X)$ can be integrated over the manifold:

$$
\int_{X} \psi=\int_{X} d x^{1} \ldots d x^{m} \int d \Theta^{1} \ldots d \Theta^{m} \psi(x, \Theta),
$$

with the integral over the Grassmann variables understood in the Berezin sense [57]. The Berezin integral is completely determined by the following set of rules:

$$
\begin{aligned}
\int d \Theta^{i} & =0, & \int \Theta^{i} d \Theta^{i} & =1, \\
\Theta^{i} d \Theta^{j} & =-d \Theta^{j} \Theta^{i}, & d \Theta^{i} d \Theta^{j} & =-d \Theta^{j} d \Theta^{i} .
\end{aligned}
$$

The integral in the r.h.s. of Eq. (19) is well defined, since it does not depend on the choice of local coordinates (the Jacobians that describe the transformations of the boson $d x$ and fermion $d \Theta$ measures under a coordinate transformation cancel each other). Note that only the maximalfermion component $\psi^{(m)}(x, \Theta)$ of $\psi(x, \Theta)$ contributes to the integral.

The above integration construction combined with the pull-back allows a fermion state $\psi \in A^{\bullet}(X)$ to be integrated over a cycle or chain $\gamma: N \rightarrow X$, that corresponds 
to the case of a manifold or manifold with a border, respectively:

$$
\int_{\gamma} \psi=\int_{N} \gamma^{*} \psi
$$

with the non-zero contribution provided by the $\psi^{(n)}$ component only.

Stokes' theorem in the case of smooth chains implies the following: for a chain $\gamma: N \rightarrow X$ and fermion state $\psi \in A^{\bullet}(X)$ we have

$$
\int_{\gamma} \mathcal{Q} \psi=\int_{\left.\gamma\right|_{\partial N}} \psi
$$

The Hodge star transformation [36], adopted to the language of fermion states, $*: A^{\bullet}(X) \rightarrow A^{m-\bullet}(X)$ associates with an $n$-fermion state $\psi^{(n)}$ an $(m-n)$-fermion state denoted by $* \psi$, and therefore $* \psi$ provides a hole description of the original fermion state $\psi$. The metric $g$ generates a standard scalar product of the states

$$
\begin{aligned}
\left(\psi^{(n)}, \varphi^{(n)}\right) & =\int_{X} \frac{d x}{\sqrt{g(x)}} n ! g^{i_{1} j_{1}}(x) \cdots g^{i_{n} j_{n}}(x) \psi_{i_{1} \cdots i_{n}}^{(n)} \varphi_{j_{1} \cdots j_{n}}^{(n)} \\
& =\int \psi^{(n)}\left(* \varphi^{(n)}\right)
\end{aligned}
$$

with the second equality being in fact the defining property of the Hodge star operator.

The Supersymmetric Fokker-Planck (SFP) operator can be presented in a very simple form in the case of an arbitrary metric $g^{i j}(x)$ in the following way. We start with introducing the force $f_{i}(x ; t)=g_{i j}(x) u^{j}(x ; t)$ that corresponds to the velocity field in the Langevin equation, and further its supersymmetric counterpart $F(x, \Theta ; t)=$ $f_{j}(x ; t) \Theta^{j}$. The SFP operator has a form

$$
\mathcal{L}=\kappa\left(\mathcal{Q} \mathcal{Q}_{F}^{\dagger}+\mathcal{Q}_{F}^{\dagger} \mathcal{Q}\right), \quad \mathcal{Q}_{F}=\mathcal{Q}+\kappa^{-1} F
$$

with Hermitian conjugation defined using an obvious scalar product in the space of states with given fermion number (or equivalently differential forms of given rank). This is the only source of dependence of the operator $\mathcal{L}$ on the metric. Several comments are in place. First, the operator $\mathcal{L}$ preserves the fermion number, also referred to as the degree, and hence so does the evolution governed by the SFP equation. Second, $[\mathcal{Q}, \mathcal{L}]$, and therefore the SFP equation preserves closed and exact states. This allows the dynamics to be restricted to closed states, with the SFP equation written in a form

$$
\frac{\partial \varrho}{\partial t}=-\mathcal{Q} J, \quad J=-\kappa \mathcal{Q}_{F}^{\dagger} \varrho
$$

of a continuity condition, as it happens in the case of the standard FP equation. One might think about the higherdimensional counterpart, defined by Eq. (25), as a suitable candidate for an average (over the stochastic process) higher-dimensional current density, in terms of which the average fluxes can be expressed. The simple relations between the higher-dimensional fluxes and current densities will be stated in section 2.5, with the derivations briefly sketched in section 3 . Finally we note that in a particular case $F=0$, the SFP operator in Eq. (24) adopts the form of the Hodge-Laplace operator, widely used in Hodge theory, whereas in a particular case of flat metric in $\mathbb{R}^{m}$ and potential force $f_{j}=-\partial_{j} V$, the operator in Eq. (25) reduces to the $\mathrm{SFP}$, considered in [74].

\subsection{Poincaré Duality and Higher-Dimensional Fluxes Generated in Stationary and Periodically Driven Sys- tems}

The relation between the higher-dimensional current densities [Eq. (25)] and generated fluxes is naturally formulated in terms of a version of Poincaré duality that involves de Rham cohomology [36], defined in section 2.4 which can be formulated as follows. Poincaré duality consists of the set of isomorphisms

$$
H_{k}(X ; \mathbb{R}) \cong H_{\mathrm{DR}}^{m-k}(X), \quad k=0, \ldots, m,
$$

naturally defined using the two pairings

$$
\begin{array}{r}
\langle\cdot, \cdot\rangle: H_{k}(X ; \mathbb{R}) \otimes H_{\mathrm{DR}}^{k}(X) \rightarrow \mathbb{R}, \\
\langle[\gamma],[\omega]\rangle=\int_{N} \gamma^{*}(\omega), \quad \gamma: N \rightarrow X,
\end{array}
$$

and

$$
\begin{gathered}
\langle\cdot, \cdot\rangle: H_{\mathrm{DR}}^{m-k}(X) \otimes H_{\mathrm{DR}}^{k}(X) \rightarrow \mathbb{R} \\
\langle[\psi],[\omega]\rangle=\int_{X} \psi \omega, \omega \in Z^{k}(X), \psi \in Z^{m-k}(X) .
\end{gathered}
$$

We will show in section 3 that a probability distribution $\mathcal{P}(\gamma)$ of $(k-1)$-dimensional cycles $\gamma: N \rightarrow X$ allows a reduced description in terms of a closed state $\varrho \in Z^{m-(k-1)}(X)$, with $[\varrho] \in H_{\mathrm{DR}}^{m-(k-1)}$ corresponding to $[\gamma] \in H_{k-1}(X)$ via Poincaré duality [Eq. (26)], that satisfies the SFP equation [Eq. (25)]. Let $\alpha: K \rightarrow X$ be an $(m-k)$-dimensional cross-section cycle. It will be shown that the average flux $\omega(\alpha ; t)$ through the cross section $\alpha$, understood as the intersection index of a stochastic trajectory with $\alpha$ per unit time, averaged over the stochastic process can be represented in a form

$$
\omega(\alpha ; t)=-\kappa t^{-1} \int_{0}^{t} d \tau \int_{K} \alpha^{*} \mathcal{Q}_{F(\tau)}^{\dagger} \varrho(\tau) .
$$

The expression for the flux adopts a specially simple form for the long-time limit in the cases of stationary and periodic driving. We start with a stationary case, when $F(\tau)=F$, so that at $t \rightarrow \infty$ we can replace $\varrho(\tau)$ with the stationary solution of the SFP equation $\varrho([\gamma])$ whose cohomology class $[\varrho]$ is Poincaré dual to the homology class $[\gamma]$ of the cycle $\gamma$ that participates in stochastic dynamics. The average flux becomes time-independent and can be represented in the form

$$
\begin{aligned}
\omega(\alpha ;[\gamma]) & =\int_{K} \alpha^{*} \omega([\gamma]), \\
\omega([\gamma]) & =-\kappa \mathcal{Q}_{F}^{\dagger} \varrho([\gamma]), \\
\mathcal{Q} \omega & =0
\end{aligned}
$$


The last condition implies $\omega([\gamma]) \in Z^{m-k}(X)$ is closed, so that taking the cohomology class $[\omega]$ defines a flux map

$$
\omega: H_{k-1}(X) \rightarrow H_{k}(X ; \mathbb{R}),
$$

which sends the homology class $[\gamma]$ of the moving cycle $\gamma$ to the homology class dual to the cohomology class $[\omega([\gamma])]$ via Poincaré duality [Eq. (26)]. It is important to note that the long-time limit of the average flux turns out to be represented by a closed state $\omega([\gamma])$, which means that the flux through a cross section $\alpha$ depends on its homology class $[\alpha]$ only, as if the trajectories were closed. As briefly discussed at the end of subsection 2.2, this is interpreted as at the long-time limit the stochastic trajectories are statistically closed.

In the periodic driving case, following [21], we consider the driving force to be represented by a potential $f_{j}(t)=$ $-\partial_{j} V(t)$ that depends on time periodically $V(t+T)=$ $V(t)$. In the periodic driving case it is advantageous to define the flux as the intersection index per one diving protocol, rather than per unit time. The expression for the average flux $\omega(\alpha,[\gamma])$ has the form of Eq. (30) with the integrand $\omega([\gamma])$ replaced with

$$
\omega([\gamma])=\int_{0}^{T} d \tau\left(-\kappa \mathcal{Q}_{F}^{\dagger}\right) \varrho([\gamma] ; \tau)
$$

with $\varrho([\gamma] ; \tau)$ being the unique closed solution of the SFP equation [Eq. (25)] with $F=-\mathcal{Q} V$ and $[\varrho([\gamma] ; \tau)]=[\gamma]$.

In section 3 , we present a derivation that starts with Eq. (32) and allows the average flux to be expressed in terms of a higher-dimensional version of the Kirchhoff problem in the continuous (rather than discrete) setting. The aforementioned Kirchhoff problem can be formulated as follows. We first note that we have an onto linear map $\mathcal{Q}: A^{m-k}(X) \rightarrow B^{m-k+1}(X)$, by definition. An attempt to invert it faces the problem of ambiguity, since the map has a kernel, which consists of the subspace $Z^{m-k}(X)$ of closed states. The uncertainty can be fixed by requiring that the image of the inverse map is orthogonal to the kernel. The above requirement allows to define a unique inverse, known in linear algebra as pseudo-inverse, as long as a scalar product in the space $A^{m-k}(X)$ of $(m-k)$ fermion states is chosen.

Consider a modified scalar product in the space $A^{m-k}(X)$, defined by

$$
(\alpha, \beta)_{V, \kappa}=\left(e^{\kappa^{-1} V} \alpha, \beta\right)=\left(\alpha, e^{\kappa^{-1} V} \beta\right),
$$

and let $A_{V, \kappa}$ be the pseudo-inverse of $\mathcal{Q}$ with respect to the modified scalar product [Eq. (33)], i.e., it is completely identified by its properties

$$
A_{V, \kappa} \mathcal{Q} \varphi=\varphi, \quad\left(A_{V, \kappa} \varphi, \psi\right)_{V, \kappa}=0,
$$

for any $\varphi \in B^{m-k+1}(X)$, and any $\psi \in Z^{m-k}(X)$. The problem of finding the pseudo-inverse in the above setting is hereafter referred to as the higher-dimensional continuous Kirchhoff problem.
In section 3 we will derive the following expression for the average flux in terms of the pseudo-inverse of $\mathcal{Q}$

$$
\omega([\gamma])=-\int_{0}^{T} d \tau A_{V(\tau), \kappa} \dot{\varrho}([\gamma] ; \tau) .
$$

where $\dot{\varrho}=d \varrho / d \tau$ denotes the time derivative. Note that $\dot{\varrho}=\mathcal{L} \varrho=\mathcal{Q}\left(\kappa \mathcal{Q}_{-\mathcal{Q} V}^{\dagger}\right)$ is closed, so that applying the pseudo-inverse $A_{V, \kappa}$ to $\dot{\varrho}$ in the r.h.s. of Eq. (35) is legitimate. Also note that applying $\mathcal{Q}$ to Eq. (35) yields, due to the first property in Eq. (34), in the r.h.s. just the time integral of $\dot{\varrho}$ over a period, which is zero. Therefore, $\omega([\gamma])$ is exact, so that the same arguments, as presented earlier in the context of stationary driving, bring us to a well-defined flux map of the same form of Eq. (31).

Finally we note that in the case of slow driving (adiabatic limit) the periodic solution of the SFP equation is well approximated $\varrho([\gamma] ; \tau)=\varrho_{\mathrm{B}}([\gamma] ; V(\tau), \kappa)$, with $\varrho_{\mathrm{B}}([\gamma] ; V, \kappa)$ hereafter, with some minimal abuse of terminology, being referred to as a higher-dimensional Boltzmann distribution. It is formally defined as a unique closed stationary solution of the SFP equation with $F=-\mathcal{Q} V$ and $\left[\varrho_{\mathrm{B}}([\gamma])\right]$ being Poincaré dual to $[\gamma]$. So in the adiabatic limit we have explicitly

$$
\omega([\gamma])=-\int_{0}^{T} d \tau A_{V(\tau), \kappa} \frac{d}{d \tau} \varrho_{\mathrm{B}}([\gamma] ; V(\tau), \kappa) .
$$

\section{Langevin Processes, Higher-Dimensional Cur- rents, and Supersymmetric Fokker-Planck Dy- namics}

In this section, we develop an approach that allows us to derive the expressions [Eqs. (29), (30), and (35)] for the average higher-dimensional current in terms of the boson/fermion states, introduced in section 2.4, and their evolution via the SFP equation. Our approach is based on introducing the reduced probability distributions or reduced measures that are much simpler objects compared to probability measures in the infinite-dimensional spaces of cycles. These evolve according to the SFP equation and contain all relevant information about the average higherdimensional fluxes.

\subsection{Generating Functions for Empirical Current Distri- butions}

Probability distributions are efficiently studied by considering the associated generating functions. It is convenient to replace the generating function, given by Eq. (5), with a more general $Z(\mathcal{A} ; \gamma, t)$, whose argument is an $k$ fermion state $\mathcal{A} \in A^{k}(X)$

$$
\begin{aligned}
Z(\mathcal{A} ; \gamma, t) & =\left\langle\exp \left(\int_{N \times[0, t]} \eta^{*}(\xi, \gamma) \mathcal{A}\right)\right\rangle_{\eta} \\
& =\int \mathcal{D} \xi e^{-\kappa^{-1} S(\xi)} \exp \left(\int_{N \times[0, t]} \eta^{*}(\xi, \gamma) \mathcal{A}\right)
\end{aligned}
$$


where, same as in Eq. (5), $S(\xi)$ is a quadratic function of the random vector field that produces the proper correlation functions of the latter [Eq. (1)], and the generating function naturally depends on the initial cycle $\gamma \cdot{ }^{7}$ We reiterate that according to the arguments presented above, the path-integral is properly and completely (i.e., in the time and configuration space domains) regularized.

Being focused on average currents we expand the generating function [Eq. (37)] to linear terms in its argument

$$
\begin{aligned}
Z(\mathcal{A} ; \gamma, t) & =1+\int_{0}^{t} d \tau \int_{X} \mathcal{A} J(\tau ; \gamma)+O\left(\mathcal{A}^{2}\right) \\
& =1+\int \mathcal{D} \xi e^{-\kappa^{-1} S(\xi)} \int_{N \times[0, t]} \eta^{*}(\xi, \gamma) \mathcal{A}+O\left(\mathcal{A}^{2}\right)
\end{aligned}
$$

where $J(\tau ; \gamma) \in A^{m-k}(X)$ is just a functional coefficient in the above linear expansion, represented by a timedependent $(m-k)$-fermion state that also depends parameterically on the initial cycle $\gamma$.

If the stochastic trajectories were closed (periodic in time), i.e., the corresponding smooth maps $\eta: N \times[0, t] \rightarrow$ $X$ could be considered as cycles $N \times S^{1} \rightarrow X$, then replacing $\mathcal{A}$ with $\mathcal{A}+\mathcal{Q B}$, referred to as a gauge transformation of $\mathcal{A}$, would not change the $\mathcal{A}$-dependent exponential term in Eq. (37) due to the Stokes' theorem (see subsection 2.4) and the generating function would be gauge invariant. Therefore, it makes sense to refer to a situation when the generating function becomes gaugeinvariant, i.e., $Z(\mathcal{A}+\mathcal{Q B})=Z(\mathcal{A})$ at long times as "at long times the trajectories are closed statistically". The referred situation can be described as follows. At long times the generating function has a large deviation form $Z(\mathcal{A} ; \gamma, t) \sim e^{-t \mathcal{F}(\mathcal{A} ;[\gamma])}$ with $\mathcal{F}(\mathcal{A} ;[\gamma])$ being gauge invariant and depending on the initial cycle $\gamma$ via its homology class $[\gamma]$. In the gauge-invariant case, the corresponding Cramér function $\mathcal{S}(J ; \gamma])$, with $J \in A^{m-n-1}(X)$ is supported by the conserving $\mathcal{Q} J=0$ currents and can be obtained from gauge-invariant $\mathcal{F}(\mathcal{A} ;[\gamma])$ by applying a standard Legendre transformation.

In the case when we restrict ourselves to closed $\mathcal{Q} \mathcal{A}=0$ fermion states for the argument of the generating function, corresponding to empirical currents $\omega=[J]$ rather than current densities, the generating function depends on the cohomology class $[\mathcal{A}] \in H^{k}(X)$. The corresponding Cramér function $\mathcal{S}(\omega ; \gamma])$ that depends on $\omega=[J] \in$ $H^{m-k}(X) \cong H_{k}(X ; \mathbb{R})$, rather than the current density $J \in Z^{m-k}(X) \subset A^{m-k}(X)$, can be obtained from $\mathcal{F}([\mathcal{A}] ;[\gamma])$ via a standard Legendre transformation.

It is worth to emphasize that, as opposed to the standard $n=0$ case, in higher dimensions gauge invariance is not guaranteed, but is rather a statistical property. Therefore, it can be broken, which means that the boundaries of the stochastic trajectories $\eta$ start playing an important

\footnotetext{
${ }^{7}$ Also note that the expression in the second exponent in Eq. (37) is just a properly defined integral $\int_{\eta} \mathcal{A}=\int_{N \times[0, t]} \eta^{*} \mathcal{A}$ of the $k$ fermion state $\mathcal{A}$ over the $k$-dimensional surface $\eta$.
}

role. We will demonstrate in this manuscript that gauge invariance is maintained at least on the level of average values of current distributions. An interesting open question is whether gauge invariance can be broken for large enough deviations of the observed current $\omega$ from its stationary value. As a first step, gauge symmetry breaking can be studied on the level of Markov-chain reduced models described in the second manuscript.

Summarizing, to demonstrate the validity of the representation, given by Eq. (29) it is enough to show that

$$
\begin{array}{r}
\int \mathcal{D} \xi e^{-\kappa^{-1} S(\xi)} \int_{N \times[0, t]} \eta^{*}(\xi, \gamma) \mathcal{A} \\
=-\kappa \int_{0}^{t} d \tau \int_{X} \mathcal{A} \mathcal{Q}_{F(\tau)}^{\dagger} \varrho(\tau)
\end{array}
$$

where $\varrho(\tau)$ is the solution of the SFP equation. This will be done in section 3.2 by introducing and studying the properties of reduced measures.

\subsection{Reduced Measures, Supersymmetric Fokker-Planck Equation and Average Higher-Dimensional Currents}

We can interpret Eq. (38) as a path-integral representation of the average current density $J$. However, path integrals are difficult to calculate. A standard trick used for Langevin processes is to switch from the Lagrangian, i.e., path-integral, picture to an equivalent, yet different representation, often referred to as the Euler or Hamilton picture. This transformation replaces a path integral by a deterministic linear equation for the relevant distributions. We refer to the obtained equation as the twisted Fokker-Planck (FP) equation, where the term "twisted" appreciates the fact that the FP equation is modified by introducing the gauge field $\mathcal{A}$ that serves as the argument of the generating function. Since in the higher-dimensional $n>1$ case, the distributions $\mathcal{P}$ are defined in the infinitedimensional space $X^{N}$ of smooth maps $N \rightarrow X$, it is absolutely imperative to view a distribution as an integration measure. That is, as a linear functional that associates with a function $h: X^{N} \rightarrow \mathbb{R}$, a number $\mathcal{P}(h)$ referred to as the integral of $h$ with respect to the measure $\mathcal{P}$; a standard notation is often used:

$$
\mathcal{P}(h)=\int_{X^{N}} d \mathcal{P}(\zeta) h(\zeta)
$$

To perform the desired transformation to the EulerHamilton picture we make use of the fact that a Langevin process can be viewed as a theory of stochastic flows, as described in some detail in section 2.1. A flow in $X$ generates a flow in the cycle space $X^{N}$ and further in the space of measures. Denoting by $\mathcal{P}(\gamma ; t)$ the value at time $t$ of the measure, with the initial condition $P(\gamma ; 0)(h)=h(\gamma)$, averaged over stochastic flows (or equivalently vector fields) we arrive at the following path-integral representation

$$
\mathcal{P}(\gamma ; t)(h)=\int \mathcal{D} \xi e^{-\kappa^{-1} S(\xi)} h(\eta(\xi, \gamma)(t)),
$$


where, according to our notation $\eta(\xi, \gamma)(t) \in X^{N}$ is the $k$-cycle that represents the end-point of the stochastic trajectory $\eta(\xi, \gamma)$ that starts at $\gamma \in X^{N}$ and is generated by the noise realization $\xi$.

The family $\mathcal{P}(\gamma ; t)$ of measures obviously satisfies the semi-group relation

$$
\mathcal{P}\left(\gamma, t+t^{\prime}\right)=\int_{X^{N}} d \mathcal{P}(\zeta ; \gamma ; t) \mathcal{P}\left(\zeta ; t^{\prime}\right)
$$

which generally serves as a starting point for deriving the corresponding FP equation by setting $t^{\prime}=\varepsilon$, followed by implementing the limit $\varepsilon \rightarrow 0$. However, as opposed to the standard $k=1$ case, the higher-dimensional situation brings an additional problem: The measure $\mathcal{P}(\gamma ; t)$, which obviously depends on the regularization, does not have a well-defined $\varepsilon \rightarrow 0$ limit. This is easy to see, since the regularized path integral in Eq. (41) contains $(\operatorname{dim} \mathcal{U})^{t / \varepsilon}$ integrations and therefore the regularized $\mathcal{P}$ is supported by a finite-dimensional surface in $X^{N}$, whose dimension $(\operatorname{dim} \mathcal{U})^{t / \varepsilon}$ grows rapidly with $\varepsilon \rightarrow 0$. We address the problem by introducing the reduced measures that can be viewed as restrictions of $\mathcal{P}$ to narrower classes of functions to be integrated.

The simplest reduction leads to simple reduced measures that allow average currents to be handled, and, therefore, this is the only reduction that is considered in this manuscript. Given a measure $\mathcal{P}$ in $X^{N}$, understood in the sense of Eq. (40) we restrict it to a vector subspace of functions $h_{\alpha}(\gamma)$, parameterized by $(k-1)$-fermion states $\alpha \in A^{k-1}(X)$ of the form

$$
h_{\alpha}(\gamma)=\int_{\gamma} \alpha=\int_{N} \gamma^{*} \alpha
$$

The reduced measure, associated with $\mathcal{P}$, is described by a closed $(n-k+1)$-fermion state, denoted $\varrho(\mathcal{P})$, completely described by the following conditions

$$
\mathcal{Q} \varrho(\mathcal{P})=0, \quad \int_{X^{N}} d \mathcal{P}(\gamma) \int_{N} \gamma^{*} \alpha=\int_{X} \varrho(\mathcal{P}) \alpha
$$

for all $\alpha \in A^{k-1}(X)$. Diffeomorphisms act on the states $\varrho$ by means of pull-backs, therefore, flows act on these states in a well-defined way, and by the construction [Eq. (44)] the reduction commutes with the flow, i.e.,

$$
\varrho(\mathcal{P}(t))=\varrho(\mathcal{P})(t)
$$

which simply means that the evolution of reduced measures can be obtained by looking at the evolution of the corresponding representing super-states $\varrho(\mathcal{P})$.

Since vector fields can be viewed as infinitesimal diffeomorphisms, the action of vector fields on the states $\varrho$ is easily identified and is known to be given by the Lie derivative of $\varrho$ with respect to a vector field $\eta$

$$
L_{\eta} \varrho=\mathcal{Q} i_{\eta} \varrho+i_{\eta} \mathcal{Q} \varrho
$$

with the inner derivative $i_{\eta}$ defined in section 2.4. To identify the evolution of a super-state we consider a short time interval $(t, t+\varepsilon)$, at which the stochastic vector field is considered to be time-independent according to our time regularization scheme, and neglect the time dependence of the velocity field, which results in

$$
\begin{aligned}
\varrho(t+\varepsilon) & \approx\left\langle\exp \left(\varepsilon L_{u(t)+\xi} \varrho(t)\right\rangle_{\xi}\right. \\
& \approx \varrho(t)+\varepsilon \mathcal{L}(t) \varrho(t)+O\left(\varepsilon^{2}\right),
\end{aligned}
$$

with the second approximate equality being just a definition of the evolution operator $\mathcal{L}(t)$. Averaging over the random vector field is performed by regularization of the space of allowed vector fields, restricting them to a finite-dimensional space, spanned on a set of vector fields $\left(e_{a} \mid a=1, \ldots, N\right)$, i.e., representing the stochastic field in the form

$$
\begin{array}{r}
\xi^{j}(x)=\sum_{a=1}^{n} \lambda_{a} e_{a}^{j}(x), \\
\left\langle\lambda_{a}\right\rangle=0, \quad\left\langle\lambda_{a} \lambda_{b}\right\rangle=2 \kappa \varepsilon^{-1} \delta_{a b}, \\
G^{i j}(x, y)=\sum_{a=1}^{N} e_{a}^{i}(x) e_{a}^{j}(y)
\end{array}
$$

Expanding the expectation value in Eq. (47) to first order in $u$ and second order in $\xi$ (to keep all terms up to order $\epsilon$ after averaging), substituting Eq. (48) into Eq. (47) performing averaging explicitly, and comparing the terms of first order in $\varepsilon$ we arrive at

$$
\mathcal{L}(t) \varrho(t)=L_{u(t)} \varrho(t)+\kappa \sum_{a=1}^{N} L_{e_{a}} L_{e_{a}} \varrho(t) .
$$

Recasting the Lie derivatives in Eq. (49) in an explicit form, and after some straightforward algebra we identify the operator $\mathcal{L}$ with the SFP operator given by Eq. (24). ${ }^{8}$ At this point we want to emphasize that the result, we have obtained, is not only crucial in deriving Eq. (39), which will be completed in the rest of this section, but also provides an important insight on the SFP equation, interpreting the latter as the dynamical equation that describes evolution of reduced measures, associated with stochastic motion of higher-dimensional cycles under a Langevin process.

We complete this section with deriving Eq. (39). With the machinery developed earlier in this section this becomes a simple and straightforward task. Denoting by $I(t ; \varepsilon)$ the contribution to the l.h.s. of Eq. (39) that is due to the fragment of a stochastic trajectory on the time interval $(t, t+\varepsilon)$, where, according to the chosen time regularization, the stochastic field is approximated by a time-

\footnotetext{
${ }^{8}$ To be precise, we should note that, similar to the standard FP operator case, the force field $F$, obtained in the above derivation contains additional terms, generated by the random field, which have the form of products of the fields $e_{a}$ and their gradients, and which can be expressed in terms of the covariant derivatives of $G^{i j}(x, y)$ with respect to $y$, taken at $y=x$.
} 
independent one we obtain

$$
\begin{aligned}
I(t ; \varepsilon) & \int_{0}^{\varepsilon} d \tau\left\langle\int_{X^{N}} d(\exp (\tau \pi(u(t)+\xi)) \mathcal{P}(t))\right. \\
& \times(\gamma) \int_{\gamma} i_{u(t)+\xi \mathcal{A}\rangle_{\xi}} \\
= & \int_{0}^{\varepsilon} d \tau \int_{X}\left\langle\varrho(\exp (\tau \pi(u(t)+\xi)) \mathcal{P}(t)) i_{u(t)+\xi} \mathcal{A}\right\rangle_{\xi} \\
= & \int_{0}^{\varepsilon} d \tau \int_{X}\left\langle\exp \left(\tau L_{u(t)+\xi} \varrho(t) i_{u(t)+\xi} \mathcal{A}\right\rangle_{\xi} .\right.
\end{aligned}
$$

where $\pi(\eta)$ denotes the action of the vector field $\eta$ on the space of measures on $X^{N}$. The first equality is due to the path-integral representation for the measure evolution [Eq. (41)] and the semigroup property [Eq. (42)], the second equality follows from the defining property of reduction [Eq. (44)], whereas the third equality reflects compatibility of the reduction with stochastic evolution [Eq. (45)]. Expanding the exponent in the most r.h.s. of Eq. (50) and further performing the averaging in the same way it was done in deriving Eq. (49) we arrive at

$$
\begin{aligned}
I(t ; \varepsilon) & \approx \varepsilon \int_{X}\left\langle\varrho(t)\left(i_{u(t)} \mathcal{A}\right)+\left(L_{\xi} \varrho\right)\left(i_{\xi} \mathcal{A}\right)\right\rangle \\
& =\varepsilon \int_{X} \varrho(t)\left(i_{u(t)} \mathcal{A}\right)+\frac{\varepsilon \kappa}{2} \sum_{a=1}^{N} \int_{X}\left(L_{e_{a}} \varrho\right)\left(i_{e_{a}} \mathcal{A}\right),
\end{aligned}
$$

and after some straightforward transformations we obtain

$$
I(t ; \varepsilon) \approx-\varepsilon \kappa \int_{X} \mathcal{A} \mathcal{Q}_{F(t)}^{\dagger} \varrho(t)
$$

Performing the summation of the contributions $I(t ; \varepsilon)$, taking the limit $\varepsilon \rightarrow 0$, combined with applying Eq. (52) immediately results in Eq. (39).

\subsection{Explicit Expression for Higher-Dimensional Cur- rents, Generated in a Stationary and Periodically- Driven System}

The expression for the stationary case flux [Eq. (30)], as shown earlier in section 2.5 follows immediately from the general expression for the generated flux in the timedependent case [Eq. (29)]; the latter as derived in sections 3.1 and 3.2. The expression for the periodic driving [Eq. (32)] also follows from Eq. (29) in a straightforward way. In this section we demonstrate how a more convenient expression for the flux [Eq. (35)] follows from Eq. (32). In fact we will demonstrate a more general property, i.e. that the relation

$$
\int_{0}^{t} d \tau\left(-\kappa \mathcal{Q}_{-\mathcal{Q} V}^{\dagger} \varrho([\gamma] ; \tau)=-\int_{0}^{t} d \tau A_{V(\tau), \kappa} \dot{\varrho}([\gamma] ; \tau)\right.
$$

holds in a general time-dependent case. This will be demonstrated by deriving the relation

$$
\kappa \mathcal{Q}_{-\mathcal{Q} V}^{\dagger} \varrho([\gamma] ; \tau)=A_{V(\tau), \kappa} \dot{\varrho}([\gamma] ; \tau) .
$$

To that end we recast $\mathcal{Q}_{-\mathcal{Q} V}=e^{\kappa^{-1} V} \mathcal{Q} e^{-\kappa^{-1} V}$, which implies $\mathcal{Q}_{-\mathcal{Q} V}^{\dagger}=e^{-\kappa^{-1} V} \mathcal{Q}^{\dagger} e^{\kappa^{-1} V}$ and allows Eq. (54) to be recast in the form

$$
\kappa e^{-\kappa^{-1} V} \mathcal{Q}^{\dagger} e^{\kappa^{-1} V} \varrho([\gamma] ; \tau)=A_{V(\tau), \kappa} \dot{\varrho}([\gamma] ; \tau) .
$$

Due to uniqueness of the pseudo-inverse (for a fixed scalar product), to show the validity Of Eq. (55) it is enough to verify that its l.h.s. satisfies the defining properties of the pseudo-inverse [Eq. (34)]. We have for the first property

$$
\mathcal{Q} \kappa e^{-\kappa^{-1} V} \mathcal{Q}^{\dagger} e^{\kappa^{-1} V} \varrho([\gamma] ; \tau)=\mathcal{L} \varrho([\gamma] ; \tau)=\dot{\varrho}([\gamma] ; \tau)
$$

and for the second one

$$
\begin{aligned}
\left(e^{-\kappa^{-1} V} \mathcal{Q}^{\dagger} e^{\kappa^{-1} V} \varrho, \psi\right)_{V, \kappa} & =\left(\mathcal{Q}^{\dagger} e^{\kappa^{-1} V} \varrho, \psi\right) \\
& =\left(e^{\kappa^{-1} V} \varrho, \mathcal{Q} \psi\right) \\
& =0
\end{aligned}
$$

since $\mathcal{Q} \psi=0$, which completes the derivation.

\section{Discussion}

In this manuscript, we have extended the concept of currents and fluxes generated in non-equilibrium (driven) stochastic processes to higher dimensions. We have done this in the continuous case, where the higherdimensional currents characterize the same process as standard stochastic currents, i.e., Langevin stochastic dynamics on a manifold $X$ (of arbitrary dimension $\operatorname{dim}(X)=$ $m$ ) with inhomogeneous noise. This has been achieved by applying the following key steps.

(i) We considered a Langevin process the way it should be considered, i.e., as a process that involves deterministic, as well as random components of the velocity field, referred to as $u^{j}(x, t)$ and $\xi^{j}(x, t)$, respectively. This is a natural view if one interprets a Langevin process as a result of eliminating fast environmental (bath) degrees of freedom, so that the random component of the velocity field is generated by the force field that describes the system-bath interaction, and further applying the overdamped limit. By introducing the more general quantity $G^{i j}(x, y)$ that characterizes the correlations of the random field $\xi^{j}(x, t)$ at different points, rather than its reduced counterpart $g^{i j}(x)=G^{i j}(x, x)$ that is usually involved in a Langevin equation, we can view a Langevin processes as a theory of stochastic flows or, equivalently, random walks in the space of diffeomorphisms of the underlying manifold $X$.

(ii) Such interpretation of a Langevin process allows stochastic dynamics of higher-dimensional (extended) objects to be considered; in particular one can look at what happens with $(k-1)$-dimensional cycles, for $k=0, \ldots, m-$ 1 under the random flow. Precisely, one can associate observables with $k$-dimensional trajectories spanned as a result of motion of $(k-1)$-cycles. Using an interpretation of the standard flux through a cross section as an intersection index of a 1-dimensional stochastic trajectory with an 
$(m-1)$-dimensional cross section, we provide a rigorous definition of higher-dimensional fluxes as new observables associated with a "good old" Langevin process, and also reduce computation of their statistical properties to, sometimes non-trivial, but still just technical details.

(iii) On the technical side, we generalized the Lagrangian (Langevin equation) Euler-Hamiltonian (FP equation) correspondence to treat stochastic dynamics of higher-dimensional cycles. This generalization faced some technical difficulties, e.g., the infinite-dimensional nature of the space $X^{N}$ of $(k-1)$-dimensional cycles, treated as smooth maps $\gamma: N \rightarrow X$, with $N$ being a smooth compact $(k-1)$-dimensional manifold. The aforementioned difficulties were bypassed by introducing a class of probability measures $d \mathcal{P}(\gamma)$ on the infinite-dimensional cycle space, understood as rules of integrations for functions $X^{N} \rightarrow \mathbb{R}$. These measures, although well-defined, are objects too complex to be efficiently handled. Therefore, by restricting the measures $d \mathcal{P}(\gamma)$ to narrower subspaces of functionsx to be integrated, we introduce the so-called reduced measures, represented by super-states $\varrho\left(x^{1}, \ldots, x^{m} ; \Theta^{1}, \ldots \Theta^{m}\right)$ on $X$, i.e., functions that depend on $m$ coordinates in $X$, as well as $m$ Grassmann (anticommuting) variables. Such states has been introduced in [74] in the context of supersymmetric stochastic theory that describes stochastic motion of extended objects in a nondriven case.

(iv) Also on the technical side, we have derived an evolution equation for the reduced measure $\varrho$ that turned out to be the SFP equation, presented in [74] in the equilibrium (non-driven) case. The way it has been derived is instructional. It is based on the aforementioned interpretation of a Langevin process as a random walk in the space of diffeomorphisms. Therefore, if we have any representation of the group of diffeomorphisms (or equivalently the Lie algebra of vector fields, as its infinitesimal counterpart), i.e., a vector space with a proper action of diffeomorphisms [the spaces of measures $d \mathcal{P}(\gamma)$ ) and reduced measures $\varrho$ provide good and useful examples], we can derive a FP equation in a standard fashion by applying the short-time $\varepsilon \rightarrow 0$ evolution operator determined by the action of the total vector field $u+\xi$, expand it to first-order and second-order terms in $u$ and $\xi$, respectively with further averaging over the random field, using its correlation function $G$. This means that the FP operator exists as a universal object, and the FP equation for a given representation can be obtained as an evaluation of the universal FP operator on a given representation, as, e.g., in the case of reduced measures $\varrho$. The above picture/derivation also implies an important property that the measure reduction procedure commutes with stochastic evolution. This property allowed us to derive a closed formal expression for the average value of a higherdimensional stochastic flux, generated over finite time, in terms of the solution $\varrho(t)$ of the SFP equation. This completes the Lagrangian Hamilton-Euler correspondence for higher-dimensional currents and fluxes.

(v) The aforementioned expression has been utilized to obtain analytical expressions for the average flux in the cases of stationary and periodic driving. We have introduced the higher-dimensional (supersymmetric) current density operator and expressed the average flux as the integral over the cross section [represented by a cycle $\alpha$ of complimentary dimension $(n-k)$ ] of the higher-dimensional (supersymmetric) current density $J$, obtained by applying the current density operator to the unique stationary solution $\varrho$ of the SFP equation, with the constraint that the cohomology class $[\varrho]$ corresponds to the homology class $[\gamma]$ of the moving cycle $\gamma$. We demonstrated that the flux depends only on the homology class $[\alpha]$ that is interpreted as that in the long time limit the $k$-dimensional trajectories are closed. We also showed that the average flux depends on the initial value of the cycle $\gamma$ via its homology class $[\gamma]$ only. Thus the higher-dimensional fluxes have been formulated in terms that closely resemble the situation of standard currents. We derived similar expressions for periodic driving, when the deterministic component of the force has potential character, and depends on time in a periodic fashion, the latter being the source of driving. The average flux is expressed in terms of the periodic solution of the SFP equation and the solution of the higherdimensional continuous version of the Kirchhoff problem that can be alternatively viewed as a pseudo-inverse operator to the supersymmetry operator $\mathcal{Q}$. This expression generalizes the result obtained in earlier work in the context of periodic Markov chain processes on graphs to the higher-dimensional continuous case $[17,19]$.

(vi) We identified the important role Poincaré duality plays in higher-dimensional stochastic fluxes, the former being a basic and celebrated concept in algebraic topology which establishes the equivalence of homology and cohomology in complimentary dimensions. Homology appears in our considerations in a straightforward way as equivalence classes $[\gamma],[\eta]$, and $[\alpha]$ of the initial cycle (whose homology class is stable in stochastic evolution), stochastic trajectory, and cross section. The cohomology appears in the de Rham form as the equivalence classes $[\varrho]$ and $[\mathcal{A}]$ of the reduced measures and argument of the generating functions, respectively. It is not surprising that Poincaré duality appears very naturally in dealing with fluxes, since fluxes are nothing more than averaged intersection indices of stochastic trajectories with cross sections, and an intersection index can be interpreted as an alternative view of Poincaré duality.

In this manuscript, we focused on the average values of higher-dimensional currents and fluxes. The question of the current and flux probability distributions, which in the long time limit are well described by large-deviation, also known as Cramér, functions $\mathcal{S}(J ; \gamma])$ and $\mathcal{S}(\omega ;[\gamma])[31]$. This is also known as the 2.5-level theory [4]. The problem can be treated by studying the generating function $Z(\mathcal{A} ; \gamma, t)$, defined by Eq. (37). In the case of standard currents the path integral representation can be easily converted to the Hamilton-Euler language, resulting in the socalled twisted FP equation, where all spatial derivatives 
are just elongated, as it is done in gauge theories, treating $\mathcal{A}$ (which in this case is a 1 -form, or a vector potential) as a gauge field. This leads to a problem which is not substantially more complex compared to finding the average currents. In the higher-dimensional case the situation is quite different: one cannot obtain a closed equation of the same level of complexity. The underlying reason is the extended nature of higher-dimensional cycles; formally it appears as a fact that the reduced measure $\varrho(\mathcal{P})$ does not satisfy a closed equation. Our preliminary analysis (these results will be published elsewhere) show that one can still treat $\varrho(\mathcal{P})$ as the first $p=1$ terms in the infinite hierarchy of reduced measures $\varrho_{p}(\mathcal{P})$, described by super-states on the Cartesian products $X^{\times p}$ of $p$ copies of $X$ and derive a set of SFP equations for $\varrho_{p}$ that involve $\varrho_{p+s}$ with $s=0,1,2$. However, it is promising that if one expands the generating function in powers of $\mathcal{A}$, which boils down to computing the higher moments of the flux, and if one is interested in the moments up to the $p$-th level, the hierarchy can be truncated on the $p$-th level. This implies that the higher moments can be computed within the same concept at a cost of working with higher-dimensional spaces. If the Cramér function is analytical in its argument, then knowing all moments can reproduce it for moderate values of the flux. However, even its analytical character is not established yet; whereas its behavior at very large deviations remains a completely open question.

Another possible extension, which might sound more moderate, but seems to be no less important, is to study average currents and their second moments in the vanishing noise limit $\kappa \rightarrow 0$, and try to establish stable connections between the statistical properties of the generated fluxes and the qualitative nature of the underlying deterministic dynamics. Strongly chaotic, e.g., mixing systems, are of special interest here. An interesting system that allows almost analytical treatment is the low-noise limit of a geodesic flow on a Riemann surface of genus $g \leq 2$ with constant (negative) curvature [56]. On the one hand, the system can be treated efficiently by implementing dynamical symmetry and decompositions in irreducible representations of the group $S O(2,1)$. On the other hand, its phase space, restricted to an energy shell has non-trivial homology in all dimensions, thus non-trivial fluxes occur in all possible dimensions $k=1,2,3$.

\section{Appendix A. Computation of Local Intersection Indices}

In this appendix we compute the local intersection indices for the example, considered in section 2.3, of a 3dimensional current, generated by a deterministic flow in $X=S U(2) \times S^{2}$. We start by choosing orientations on $\mathbb{R} \times N=\mathbb{R} \times S^{2}, K=S^{2}$, and $X=S U(2) \times S^{2}$. We will be formulating everything in terms of dimensionless time $\tau=k t$. Orientations of cartesian products will be given by the orientations of their components. Orientation of $\mathbb{R}$ is given by a global basis set represented by a constant vector field, determined the unit vector $e_{0}(\tau)=e_{0}$, so that differentiation with respect to this vector field is given by $\partial / \partial \tau$. Orientation of $S^{2}$ is chosen by picking at point $\boldsymbol{n}$ any basis set $\left(\boldsymbol{e}_{1}, \boldsymbol{e}_{2}\right)=(\boldsymbol{e},[\boldsymbol{n}, \boldsymbol{e}])$ with $\left(\boldsymbol{e} \cdot \boldsymbol{n}=0\right.$, and $\boldsymbol{e}^{2}=1$. Orientation of $S U(2)$ is chosen by introducing a global basis set $\left(\boldsymbol{u}_{1}(g), \boldsymbol{u}_{2}(g), \boldsymbol{u}_{2}(g)\right)$, generated by right-invariant vector fields, i.e., we set $\boldsymbol{u}_{a}(g)=i \sigma_{a} g$, for $a=1,2,3$. Note that in defining the basis sets in $S^{2}$ we made use of standard embeddings $S^{2} \subset \mathbb{R}^{3}$ and $S U(2) \cong S^{3} \subset \mathbb{R}^{4}$. We further note that, since the maps $\eta: \mathbb{R} \times S^{2} \rightarrow S U(2) \times S^{2}$ and $\alpha: S^{2} \rightarrow S U(2) \times S^{2}$ are both the identity on their $S^{2}$ components, in comparing the basis sets we can restrict ourselves to considering the map $F: \mathbb{R} \times S^{2} \rightarrow S U(2)$ that represents the first components of the map $\eta$, defined by Eq. (12)

$$
\begin{aligned}
F(\tau, \boldsymbol{n}) & =\exp (i \tau(\boldsymbol{n} \cdot \boldsymbol{\sigma})) \\
& =\cos (\tau)+i(\boldsymbol{n} \cdot \boldsymbol{\sigma}) \sin (\tau)
\end{aligned}
$$

and compare the basis sets $\left(d F(\tau, \boldsymbol{n})\left(e_{0}\right), d F(\tau, \boldsymbol{n})(\boldsymbol{e}), d F(\tau, \boldsymbol{n})([\boldsymbol{n}, \boldsymbol{e}])\right) \quad$ with $\left(\boldsymbol{u}_{1}, \boldsymbol{u}_{2}, \boldsymbol{u}_{3}\right)$. The action of the differential $d F$ is easily identified by noting that relaxing the condition $\boldsymbol{n}^{2}=1$ in Eq. (A.1) defines a map $F: \mathbb{R} \times \mathbb{R}^{3} \rightarrow \mathbb{R}^{4}$ that produces the original map $F: \mathbb{R} \times S^{2} \rightarrow S U(2)$ by means of an obvious restriction, resulting in

$$
\begin{aligned}
& d F\left(e_{0}\right)=\frac{\partial F}{\partial \tau}=-\sin (\tau)+i(\boldsymbol{n} \cdot \boldsymbol{\sigma}) \cos (\tau) \\
& d F\left(\boldsymbol{e}_{s}\right)=\frac{\partial F}{\partial \boldsymbol{n}} \cdot \boldsymbol{e}_{s}=i \boldsymbol{\sigma} \cdot \boldsymbol{e}_{s} \sin (\tau), \quad s=1,2
\end{aligned}
$$

To compare the basis set, represented by Eq. (A.2) with the right-invariant basis set in $S U(2)$, we compute $d F\left(e_{0}\right)\left(F(\tau, \boldsymbol{n})^{-1}, d F(\boldsymbol{e})(F(\tau, \boldsymbol{n}))^{-1} \in s u(2)\right.$. For the "north" intersection point we have $\tau=\pi / 2, \boldsymbol{n}=(0,0,1)$, $\boldsymbol{e}_{1}=(1,0,0), \boldsymbol{e}_{2}=(0,1,0)$. We find $F(\tau, \boldsymbol{n})=i \sigma_{3}$, $\sin (\tau)=1, \cos (\tau)=0$ and further

$$
\begin{aligned}
& d F\left(e_{0}\right) F^{-1}=-\left(i \sigma_{3}\right)^{-1}=i \sigma_{3}, \\
& d F\left(\boldsymbol{e}_{1}\right) F^{-1}=\left(i \sigma_{1}\right)\left(i \sigma_{3}\right)^{-1}=-\sigma_{3} \sigma_{1}=-i \sigma_{2}, \\
& d F\left(\boldsymbol{e}_{2}\right) F^{-1}=\left(i \sigma_{2}\right)\left(i \sigma_{3}\right)^{-1}=\sigma_{2} \sigma_{3}=i \sigma_{1},
\end{aligned}
$$

resulting in the basis set $\left(i \sigma_{3},-i \sigma_{2}, i \sigma_{1}\right)$, which has the same orientation as $\left(i \sigma_{1}, i \sigma_{2}, i \sigma_{3}\right)$. The local intersection index is +1 .

For the "south" intersection point we have $\tau=-\pi / 2$, $\boldsymbol{n}=(0,0,-1), \boldsymbol{e}_{1}=(1,0,0), \boldsymbol{e}_{2}=(0,-1,0)$. We find $F(\tau, \boldsymbol{n})=i \sigma_{3}, \sin (\tau)=-1, \cos (\tau)=0$ and further

$$
\begin{aligned}
& d F\left(e_{0}\right) F^{-1}=\left(i \sigma_{3}\right)^{-1}=-i \sigma_{3}, \\
& d F\left(\boldsymbol{e}_{1}\right) F^{-1}=-\left(i \sigma_{1}\right)\left(i \sigma_{3}\right)^{-1}=\sigma_{3} \sigma_{1}=i \sigma_{2}, \\
& d F\left(\boldsymbol{e}_{2}\right) F^{-1}=-\left(-i \sigma_{2}\right)\left(i \sigma_{3}\right)^{-1}=\sigma_{2} \sigma_{3}=i \sigma_{1},
\end{aligned}
$$

resulting in the basis set $\left(-i \sigma_{3}, i \sigma_{2}, i \sigma_{1}\right)$, which has the same orientation as $\left(i \sigma_{1}, i \sigma_{2}, i \sigma_{3}\right)$. The local intersection index is +1 . 
The obtained result can be also formulated in the following way. Due to periodicity of the map $F: \mathbb{R} \times S^{2} \rightarrow S U(2)$ it defines a map $F: S^{1} \times S^{2} \rightarrow S U(2)$; our computation shows that the degree of the latter is equal to +2 .

\section{Acknowledgements}

This material is based upon work supported by the National Science Foundation under Grant No. CHE-1111350 and Simons Foundation Collaboration Grant No. 317496

[1] David Andrieux and Pierre Gaspard. Fluctuation theorem for currents and schnakenberg network theory. Journal of statistical physics, 127(1):107-131, 2007.

[2] R. Dean Astumian. Adiabatic operation of a molecular machine. Proceedings of the National Academy of Sciences, 104(50):19715-19718, December 2007.

[3] R Dean Astumian. Design principles for brownian molecular machines: how to swim in molasses and walk in a hurricane. Physical Chemistry Chemical Physics, 9(37):5067-5083, 2007.

[4] Andre C Barato and Raphael Chetrite. A formal view on level 2.5 large deviations and fluctuation relations. Journal of Statistical Physics, 160(5):1154-1172, 2015.

[5] Lorenzo Bertini, Alberto De Sole, Davide Gabrielli, Gianni Jona-Lasinio, and Claudio Landim. Current fluctuations in stochastic lattice gases. Physical review letters, 94(3):030601, 2005.

[6] Lorenzo Bertini, Alberto De Sole, Davide Gabrielli, Giovanni Jona-Lasinio, and Claudio Landim. Non equilibrium current fluctuations in stochastic lattice gases. Journal of statistical physics, 123(2):237-276, 2006.

[7] Joris Bierkens, Vladimir Y Chernyak, Michael Chertkov, and Hilbert J Kappen. Linear pdes and eigenvalue problems corresponding to ergodic stochastic optimization problems on compact manifolds. Journal of Statistical Mechanics: Theory and Experiment, 2016(1):013206, 2016.

[8] Thierry Bodineau and Bernard Derrida. Current fluctuations in nonequilibrium diffusive systems: an additivity principle. Physical review letters, 92(18):180601, 2004.

[9] J-P Bouchaud and Rama Cont. A langevin approach to stock market fluctuations and crashes. The European Physical Journal B-Condensed Matter and Complex Systems, 6(4):543-550, 1998.

[10] Michael J Catanzaro, Vladimir Y Chernyak, and John R Klein. A higher boltzmann distribution. arXiv preprint arXiv:1506.06775, 2015.

[11] Michael J Catanzaro, Vladimir Y Chernyak, and John R Klein. Kirchhoffs theorems in higher dimensions and reidemeister torsion. Homology, Homotopy and Applications, 17(1), 2015.

[12] V Chernyak, M Chertkov, and C Jarzynski. Dynamical generalization of nonequilibrium work relation. Physical Review E, $71(2): 025102,2005$.

[13] V Chernyak, M Chertkov, I Kolokolov, and A Peleg. Outage probability for soliton transmission. EPL (Europhysics Letters), 66(4):499, 2004.

[14] Vladimir Y Chernyak, Michael Chertkov, Joris Bierkens, and Hilbert J Kappen. Stochastic optimal control as nonequilibrium statistical mechanics: calculus of variations over density and current. Journal of Physics A: Mathematical and Theoretical, 47(2):022001, 2013.

[15] Vladimir Y Chernyak, Michael Chertkov, Sergey V Malinin, and Razvan Teodorescu. Non-equilibrium thermodynamics and topology of currents. Journal of Statistical Physics, 137(1):109147,2009

[16] Vladimir Y Chernyak, Michael Chertkov, and Nikolai A Sinitsyn. The geometric universality of currents. Journal of Statistical Mechanics: Theory and Experiment, 2011(09):P09006, 2011.
[17] Vladimir Y Chernyak, John R Klein, and Nikolai A Sinitsyn. Quantization and fractional quantization of currents in periodically driven stochastic systems. i. average currents. The Journal of chemical physics, 136(15):154107, 2012.

[18] Vladimir Y Chernyak, John R Klein, and Nikolai A Sinitsyn. Quantization and fractional quantization of currents in periodically driven stochastic systems. ii. full counting statistics. The Journal of chemical physics, 136(15):154108, 2012.

[19] Vladimir Y Chernyak, John R Klein, and Nikolai A Sinitsyn. Algebraic topology and the quantization of fluctuating currents. Advances in Mathematics, 244:791-822, 2013.

[20] Vladimir Y Chernyak and NA Sinitsyn. Discrete changes of current statistics in periodically driven stochastic systems. Journal of Statistical Mechanics: Theory and Experiment, 2010(07):L07001, 2010.

[21] Vladimir Y Chernyak and Nikolai A Sinitsyn. Robust quantization of a molecular motor motion in a stochastic environment. The Journal of chemical physics, 131(18):181101, 2009.

[22] VY Chernyak and NA Sinitsyn. Pumping restriction theorem for stochastic networks. Physical review letters, 101(16):160601, 2008.

[23] M Chertkov, G Falkovich, I Kolokolov, and V Lebedev. Normal and anomalous scaling of the fourth-order correlation function of a randomly advected passive scalar. Physical Review E, 52(5):4924, 1995.

[24] Michael Chertkov, Scott Backhaus, and Vladimir Lebedev. Cascading of fluctuations in interdependent energy infrastructures: Gas-grid coupling. Applied Energy, 160:541-551, 2015.

[25] Michael Chertkov, Michael Fisher, Scott Backhaus, Russell Bent, and Sidhant Misra. Pressure fluctuations in natural gas networks caused by gas-electric coupling. In System Sciences (HICSS), 2015 48th Hawaii International Conference on, pages 2738-2747. IEEE, 2015.

[26] William T Coffey, Yu P Kalmykov, and JT Waldron. The langevin equation with applications to stochastic problems in physics, chemistry and electrical engineering second edition. World scientific series in contemporary chemical physics, 14, 1996.

[27] Gavin E Crooks. Nonequilibrium measurements of free energy differences for microscopically reversible markovian systems. Journal of Statistical Physics, 90(5-6):1481-1487, 1998.

[28] Gavin E. Crooks. Entropy production fluctuation theorem and the nonequilibrium work relation for free energy differences. Phys. Rev. E, 60:2721-2726, Sep 1999.

[29] Gavin E Crooks. Path-ensemble averages in systems driven far from equilibrium. Physical review E, 61(3):2361, 2000.

[30] Sybren Ruurds De Groot and Peter Mazur. Non-equilibrium thermodynamics. Courier Corporation, 2013.

[31] Amir Dembo and Ofer Zeitouni. Large deviations techniques and applications, volume 38. Springer Science \& Business Media, 2009.

[32] Bernard Derrida. Non-equilibrium steady states: fluctuations and large deviations of the density and of the current. Journal of Statistical Mechanics: Theory and Experiment, 2007(07):P07023, 2007.

[33] G Gallavotti and EGD Cohen. Dynamical ensembles in nonequilibrium statistical mechanics. Physical Review Letters, 74(14):2694, 1995.

[34] Cristian Giardina, Jorge Kurchan, and Luca Peliti. Direct evaluation of large-deviation functions. Physical review letters, 96(12):120603, 2006.

[35] Daniel T Gillespie. The chemical langevin equation. The Journal of Chemical Physics, 113(1):297-306, 2000.

[36] Phillip Griffiths and Joseph Harris. Principles of algebraic geometry. John Wiley \& Sons, 2014.

[37] Peter Hänggi, Peter Talkner, and Michal Borkovec. Reactionrate theory: fifty years after Kramers. Reviews of modern physics, 62(2):251, 1990.

[38] RJ Harris and GM Schütz. Fluctuation theorems for stochastic dynamics. Journal of Statistical Mechanics: Theory and Experiment, 2007(07):P07020, 2007. 
[39] Takahiro Hatano. Jarzynski equality for the transitions between nonequilibrium steady states. Physical Review E, 60(5):R5017, 1999.

[40] Takahiro Hatano and Shin-ichi Sasa. Steady-state thermodynamics of langevin systems. Physical review letters, 86(16):3463, 2001.

[41] Jordan Horowitz and Christopher Jarzynski. Comparison of work fluctuation relations. Journal of Statistical Mechanics: Theory and Experiment, 2007(11):P11002, 2007.

[42] Jordan M Horowitz and Suriyanarayanan Vaikuntanathan. Nonequilibrium detailed fluctuation theorem for repeated discrete feedback. Physical Review E, 82(6):061120, 2010.

[43] C Jarzynski. Equilibrium free energies from nonequilibrium processes. Acta Physica Polonica. Series B, 29(6):1609-1622, 1998.

[44] Christopher Jarzynski. Equilibrium free-energy differences from nonequilibrium measurements: A master-equation approach. Physical Review E, 56(5):5018, 1997.

[45] Christopher Jarzynski. Nonequilibrium equality for free energy differences. Physical Review Letters, 78(14):2690, 1997.

[46] Frank Jülicher, Armand Ajdari, and Jacques Prost. Modeling molecular motors. Reviews of Modern Physics, 69(4):1269, 1997.

[47] Hendrik Anthony Kramers. Brownian motion in a field of force and the diffusion model of chemical reactions. Physica, $7(4): 284-304,1940$.

[48] Jorge Kurchan. Fluctuation theorem for stochastic dynamics. Journal of Physics A: Mathematical and General, 31(16):3719, 1998.

[49] Min G. Lee, Akihiko Oba, and Hideki Takayasu. Parameter Estimation of a Generalized Langevin Equation of Market Price, pages 260-270. Springer Japan, Tokyo, 2002.

[50] David A Leigh, Jenny KY Wong, François Dehez, and Francesco Zerbetto. Unidirectional rotation in a mechanically interlocked molecular rotor. Nature, 424(6945):174-179, 2003.

[51] Don S Lemons and Paul Langevin. An introduction to stochastic processes in physics. JHU Press, 2002.

[52] Christian Maes and Karel Netočnỳ. Canonical structure of dynamical fluctuations in mesoscopic nonequilibrium steady states. EPL (Europhysics Letters), 82(3):30003, 2008.

[53] Christian Maes, Karel Netočnỳ, and Bram Wynants. Steady state statistics of driven diffusions. Physica A: Statistical Mechanics and its Applications, 387(12):2675-2689, 2008.

[54] Yuriy Makhlin and Alexander D. Mirlin. Counting statistics for arbitrary cycles in quantum pumps. Phys. Rev. Lett., 87:276803, Dec 2001.

[55] Sergey V Malinin and Vladimir Y Chernyak. Classical nonlinear response of a chaotic system. i. collective resonances. Physical Review E, 77(5):056201, 2008.

[56] Sergey V Malinin and Vladimir Y Chernyak. Classical nonlinear response of a chaotic system. ii. langevin dynamics and spectral decomposition. Physical Review E, 77(5):056202, 2008.

[57] Yuri I Manin. Gauge field theory and complex geometry, volume 289. Springer Science \& Business Media, 2013.

[58] Patrick Milan, Matthias Wächter, and Joachim Peinke. Stochastic modeling and performance monitoring of wind farm power production. Journal of Renewable and Sustainable Energy, 6(3):033119, 2014.

[59] John Milnor and James D Stasheff. Characteristic Classes.(AM-76), volume 76. Princeton University Press, 2016.

[60] Hiroyuki Noji, Ryohei Yasuda, Masasuke Yoshida, and Kazuhiko Kinosita. Direct observation of the rotation of f1atpase. Nature, 386(6622):299-302, 1997.

[61] Jun Ohkubo. The stochastic pump current and the nonadiabatic geometrical phase. Journal of Statistical Mechanics: Theory and Experiment, 2008(02):P02011, 2008.

[62] Bernt Oksendal. Stochastic differential equations: an introduction with applications. Springer Science \& Business Media, 2013.

[63] Juan MR Parrondo. Reversible ratchets as Brownian particles in an adiabatically changing periodic potential. Physical Review E, 57(6):7297, 1998 .
[64] Saar Rahav, Jordan Horowitz, and Christopher Jarzynski. Directed flow in non-adiabatic stochastic pumps. Physical Review Letters, 101(14), October 2008. arXiv: 0808.0015.

[65] Frederick Reif. Fundamentals of statistical and thermal physics. Waveland Press, 2009.

[66] Hannes Risken. Fokker-planck equation. In The Fokker-Planck Equation, pages 63-95. Springer, 1984.

[67] Stephen Roberts and Boris Muzykantskii. Spectral decompositions for evolution operators of mixing dynamical systems. Journal of Physics A: Mathematical and General, 33(48):8953, 2000 .

[68] Udo Seifert. Entropy production along a stochastic trajectory and an integral fluctuation theorem. Physical review letters, 95(4):040602, 2005.

[69] Jean-Pierre Serre. Lie algebras and Lie groups: 1964 lectures given at Harvard University. Springer, 2009.

[70] N. A. Sinitsyn. Stochastic pump effect and geometric phases in dissipative and stochastic systems. Journal of Physics A: Mathematical and Theoretical, 42(19):193001, May 2009. arXiv: 0903.4231 .

[71] NA Sinitsyn and Ilya Nemenman. The berry phase and the pump flux in stochastic chemical kinetics. EPL (Europhysics Letters), 77(5):58001, 2007.

[72] Nikolai A Sinitsyn, Alexei Akimov, and Vladimir Y Chernyak. Supersymmetry and fluctuation relations for currents in closed networks. Physical Review E, 83(2):021107, 2011.

[73] Edwin H Spanier. Algebraic Topology. Mc Graw-Hill, 1966.

[74] Sorin Tănase-Nicola and Jorge Kurchan. Metastable states, transitions, basins and borders at finite temperatures. Journal of Statistical Physics, 116(5-6):1201-1245, 2004.

[75] Nicolaas Godfried Van Kampen. Stochastic processes in physics and chemistry, volume 1. Elsevier, 1992. 